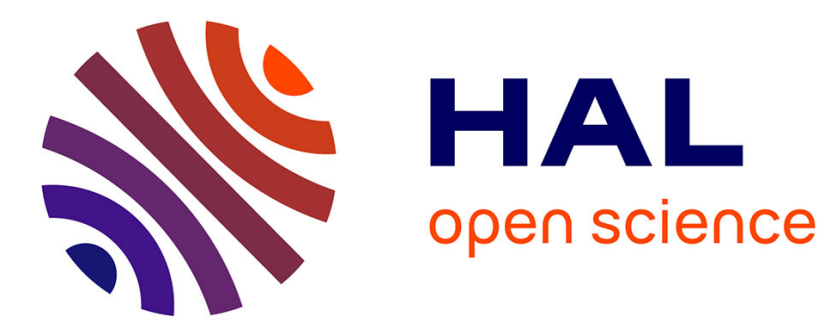

\title{
Structure investigation of nanohybrid PDMA/silica hydrogels at rest and under uniaxial deformation
}

Séverine Rose, Alba Marcellan, Tetsuharu Narita, François Boué, Fabrice Cousin, Dominique Hourdet

\section{- To cite this version:}

Séverine Rose, Alba Marcellan, Tetsuharu Narita, François Boué, Fabrice Cousin, et al.. Structure investigation of nanohybrid PDMA/silica hydrogels at rest and under uniaxial deformation. Soft Matter, 2015, 11, pp.5905-5917 10.1039/C5SM01121C . hal-01171811

\section{HAL Id: hal-01171811 \\ https://hal.sorbonne-universite.fr/hal-01171811}

Submitted on 6 Jul 2015

HAL is a multi-disciplinary open access archive for the deposit and dissemination of scientific research documents, whether they are published or not. The documents may come from teaching and research institutions in France or abroad, or from public or private research centers.
L'archive ouverte pluridisciplinaire HAL, est destinée au dépôt et à la diffusion de documents scientifiques de niveau recherche, publiés ou non, émanant des établissements d'enseignement et de recherche français ou étrangers, des laboratoires publics ou privés. 


\section{Structure investigation of nanohybrid PDMA/silica}

\section{hydrogels at rest and under uniaxial deformation.}

Séverine Rose ${ }^{1}$, Alba Marcellan ${ }^{1}$, Tetsuharu Narita ${ }^{1}$, François Boué ${ }^{2}$, Fabrice Cousin ${ }^{2}$ and Dominique Hourdet ${ }^{1} *$

${ }^{1}$ ESPCI / Sorbonne Universités, UPMC Univ Paris 06 / CNRS

UMR 7615, SIMM, 10 rue Vauquelin, F-75005, Paris, France

${ }^{2}$ Laboratoire Léon Brillouin, CEA-CNRS, Saclay 91191 Gif-sur-Yvette Cedex, France

* To whom correspondence should be addressed:

Tel. +33 (0)1 40794643 / Fax +33 (0)1 40794686

$\underline{\text { Email addresses : }}$

severine.rose@espci.fr, alba.marcellan@espci.fr, tetsuharu.narita@espci.fr, francois.boue@cea.fr, fabrice.cousin@cea.fr,dominique.hourdet@espci.fr 


\section{ABSTRACT}

Nano-hybrid hydrogels were prepared by cross-linking polymerization of $N, N$ dimethylacrylamide (DMA) within a dispersion of silica nano-particles. Working at constant polymer/water ratio, the mechanical properties of hydrogels can be finely tuned by changing either the level of covalent cross-linker and/or the amount of particles that act as physical cross-linkers through specific adsorption of PDMA chains. Whatever is the cross-linking ratio (from 0 to $1 \mathrm{~mol} \%$ ), the introduction of silica nano-particles dramatically improves the mechanical behavior of hydrogels with a concomitant increase of stiffness and nominal strain at failure. The physical interactions being reversible in nature, the dynamics of the adsorption/desorption process of PDMA chains directly controls the time-dependence of the mechanical properties. Small angle neutron scattering experiments, performed in contrast matching conditions, show that silica particles, which repel themselves at short range, remain randomly dispersed during the formation of the PDMA network. Although PDMA chains readily interact with silica particles, no significant variation of the polymer concentration was observed in the vicinity of silica surfaces. Together with the time dependence of physical interactions pointed out by mechanical analyses, this result is attributed to the moderate adsorption energy of PDMA chains with silica surfaces at $\mathrm{pH}$ 9. From 2D SANS experiments, it was shown that strain rapidly gives rise to a non affine deformation of the hybrid network with shearing due to the transverse compression of the particles. After loading at intermediate deformation, the particles recover their initial distribution due to the covalent network that is not damaged in these conditions. That is no longer true at high deformation where residual anisotropy is observed.

Keywords: gel, hybrid hydrogel, silica, poly( $N, N$-dimethylacrylamide), mechanical behavior, neutron scattering 


\section{INTRODUCTION}

Nowadays, hydrogels are used in many bio-applications ${ }^{[1-4]}$ such as superabsorbants, contact lenses, bio-sensors, drug delivery systems and scaffolds for tissue engineering. Unfortunately, the low friction level involved within swollen hydrogels under deformation and the broad distribution of polymer chain lengths between neighboring cross-links make hydrogels intrinsically brittle and poorly extensible. The reinforcement of their mechanical behavior is then a critical issue which has driven an intense activity mainly during the last ten years. In this competitive field, several strategies have been considered using improved architectures like double networks ${ }^{[5]}$, slide-ring gels ${ }^{[6]}$ or topological gels ${ }^{[7]}$ but a very versatile approach, inspired by the toughening mechanism in filled rubber materials, concerns hydrogels embedding nano-fillers; namely nanocomposite or nanohybrid hydrogels. Pioneering works in this field have been carried out by Haraguchi and co-workers ${ }^{[8-9]}$ who developed highly extensible nanocomposite gels (NC gels) by polymerizing $\mathrm{N}$-alkylacrylamide monomers in the presence of exfoliated clay platelets. These NC gels showed very high stiffness and deformability (until $1000 \%$ ) that can be tuned with their composition; mainly through the clay/polymer weight ratio as there is no chemical cross-linker added. Similarly, the addition of silica nanoparticles can strongly impact the mechanical behavior of hydrogels, either by forming covalent bonds with the polymer matrix using vinyl silane intermediate ${ }^{[10-13]}$ or by physically interacting with polymers like poly(alkylene oxide) or poly $(N \text {-alkylacrylamide })^{[14]}$. In this latter case, the use of reversible interactions between silica nanoparticles and polymer chains has been shown to dramatically enhance the dissipation process and the viscoelastic properties in aqueous media. Initially applied to the formulation of viscous fluids, prepared by gently mixing a suspension of silica nano-particles with a solution of grafted copolymers designed with adsorbing pendant stickers (PEO, PNIPAM or PDMA) ${ }^{[14-16]}$, this formulation platform was revisited to design hybrid hydrogels by polymerizing the monomer precursor of 
adsorbing chains (PDMA) directly within the silica suspension ${ }^{[17-19]}$. In this case, a small amount of chemical cross-linker can be added in order to fix the 3D topology and the mechanical properties of the material; tuning the system from a viscoelastic liquid to a viscoelastic solid $^{[18-19]}$.

In the case of hybrid hydrogels prepared by cross-linking polymerization of DMA in silica suspension, we have shown that the presence of silica nanoparticles greatly enhances the whole mechanical properties ${ }^{[17,19-20]}$. Interestingly, both initial modulus, dissipation, nominal strain at break and fracture energy were seen to be simultaneously enhanced with increasing amount of silica. As shown with weakly cross-linked systems (with level of crosslinking $\mathrm{R} \leq$ $0.1 \mathrm{~mol} \%$ ), the mechanical properties interestingly couple the elastic contribution of the organic cross-linked network with the time dependence of the physical interactions. While the intrinsic elasticity can be controlled through structural parameters like polymer concentration and degree of cross-linking, the time-dependence properties can be tuned with the silica concentration or other physicochemical parameters affecting the characteristics of the physical network like the number of physical connections and/or their lifetime. Nevertheless, while the overall organic/inorganic composition of the network controls the mechanical behaviour at the first order, the self-organization and local structure of both silica particles and polymer chains are also expected to impact the ultimate properties of the material.

Based on a wide set of nanohybrid hydrogels, differing by the level of physical and chemical cross-linkers, this work aims at investigating the local structure of these networks in relation with the reinforcement properties. After a general presentation of their mechanical properties, the main focus of this study is dedicated to the structural investigation of hybrid networks, at rest and under deformation, by small angle neutron scattering (SANS). The main issues that we want to address are the following: 
i) What is the self-organization of silica particles in the original suspension and does it change during the formulation of hybrid hydrogels and the cross-linking polymerization?

ii) Do the polymer chains, adsorbed on silica particles, form a hard layer at the silica interface?

iii) What is the origin of the reinforcement in nanohybrid hydrogels?

iv) How does the 3D structure of silica particles change under uniaxial deformation? Is this process reversible or not?

\section{EXPERIMENTAL SECTION}

\section{Materials}

$N, N$-dimethylacrylamide (DMA, 99\%, Sigma Aldrich), N,N'-methylenebisacrylamide (MBA, Fluka), potassium persulfate (KPS, Acros Organics) and $N, N, N^{\prime}, N^{\prime}-$ tetramethylethylenediamine (TEMED, 99,5\%, Sigma Aldrich) were used as received without further purification.

The silica particles (Ludox TM-50 from Dupont) were obtained from Aldrich. They were characterized in the dry state by SEM and in dilute suspensions by dynamic light scattering using a ALV/CGS-3 compact goniometer (ALV, Langen, Germany) and small angle neutron scattering (SANS). The different techniques give, respectively, an average radius $\mathrm{R}_{\mathrm{f}}=140$, 170 and $135 \AA$ for the silica particles with a specific area $S_{\text {spe }}=\left(3 / \mathrm{d}_{\mathrm{Si}} \mathrm{R}_{\mathrm{f}}\right) \cong 90 \mathrm{~m}^{2} / \mathrm{g}$, using $\mathrm{d}_{\mathrm{Si}}=2.3 \mathrm{~g} / \mathrm{cm}^{3}$ for the density of pure silica and $\mathrm{R}_{\mathrm{f}}=140 \AA$. The silica suspension $(52 \mathrm{wt} \%$; $\mathrm{pH}=9$ and conductivity $4.9 \mathrm{mS} / \mathrm{cm}$ ) was used as received for the synthesis of hybrid hydrogels. 


\section{Preparation of hybrid hydrogels}

Poly $\left(N, N\right.$-dimethylacrylamide) hybrid hydrogels were prepared at $25{ }^{\circ} \mathrm{C}$ under nitrogen atmosphere by free-radical polymerization of DMA and MBA in an aqueous suspension of silica nanoparticles using KPS and TEMED as redox initiatiors ${ }^{[17,19]}$. The gelation process takes place in home-made moulds consisting of two covered glass plates separated by a stainless steel spacer of $2 \mathrm{~mm}$ thick $\left(t_{0}\right)$. After 24 hours, hydrogels were removed from the mould and immediately characterized, or stored into paraffine oil to prevent samples from drying before analysis. For all the syntheses, the molar ratio (DMA)/(KPS)/(TEMED) was set equal to $100 / 1 / 1$ while the relative amounts of DMA, MBA, silica and water were varied, keeping constant the weight ratio Polymer/Water (PW). The nomenclature of hydrogels is SPx_PWy_Rz; with S for Silica, P for Polymer, W for Water and R for the level of crosslinking. $\mathrm{x}$ is the weight ratio between silica and polymer (varying between 0 and 5), $\mathrm{y}$ is the weight ratio between polymer and water and $\mathrm{z}$ is the MBA/DMA molar percentage with $\mathrm{z}$ $=0,0.1,1$ and up to $5 \mathrm{~mol} \%$. The following study was based on hydrogels at the preparation state: with a fixed weight ratio between polymer and water: $y=0.14$. This corresponds (in the case of $\mathrm{H}_{2} \mathrm{O}$ ) to a fixed polymer concentration equals to $12.5 \mathrm{wt} \%$ or to a fixed polymer volume fraction $\phi_{0}=0.118$.

\section{Gel composition and swelling experiments}

As previously reported, the analysis of organic and inorganic extractibles was systematically carried out on hybrid hydrogels after swelling experiments ${ }^{[19]}$. The samples, originally in their preparation state, were weighed and placed at room temperature in a large excess of solvent. The solvent was changed every day during 10 days and the swelling at equilibrium was calculated from the volume ratio between the swollen polymer network (polymer + solvent) and the dried polymer; the silica being not considered in the calculation. 
For all the samples, it was shown that the polymerization efficiency was above $98 \%$ and the amount of extractible polymer chains, when observable, was less than $1 \mathrm{wt} \%$ of the total organic content $^{[19]}$. Similarly, the silica content of hybrid hydrogels, controlled by Thermal Gravimetric Analysis, does not show any significant difference of the organic/inorganic ratio before and after swelling at equilibrium during two weeks in pure water.

\section{Small Angle Neutron Scattering (SANS)}

SANS experiments were performed at Laboratoire Léon Brillouin (CEA-Saclay, France) on spectrometers PACE and PAXY dedicated to isotropic and anisotropic measurements, respectively. Two experimental configurations were used by keeping constant the sample-todetector distance $(D=4.7 \mathrm{~m})$ and changing the wavelength of the incident neutron beam $(\lambda=12$ and $4.5 \AA$ ). These configurations provide a scattering vector modulus $q=(4 \pi / \lambda) \sin (\theta / 2)$ ranging between 0.0034 and $0.098 \AA^{-1}$ (where $\theta$ is the scattering angle).

In order to investigate the contribution of both organic and inorganic components within hybrid hydrogels, contrast matching has been carried out using suitable $\mathrm{H}_{2} \mathrm{O} / \mathrm{D}_{2} \mathrm{O}$ ratios. For that purpose, hydrogels plates were specially prepared (see supporting information) using three different aqueous environments with $\mathrm{H}_{2} \mathrm{O} / \mathrm{D}_{2} \mathrm{O}$ volume fractions $=1 / 0,0.79 / .21$ and $0.42 / 0.58$. The two last conditions were used for matching either the polymer network or the silica particles.

For isotropic analyses $(\mathrm{PACE})$, gel discs $($ diameter $=14 \mathrm{~mm}$ and thickness $=2 \mathrm{~mm})$ punched from plate samples in their preparation state, were fit inside a ring spacer hermetically sandwiched between two quartz slides and let under the neutron beam between 10 and 90 min, depending on solvent $\left(\mathrm{H}_{2} \mathrm{O} / \mathrm{D}_{2} \mathrm{O}\right)$ and silica content. For anisotropic measurements performed on PAXY (hydrogels under deformation), a special device has been developed as described in supporting information (see Figure S1). With this setup, the hydrogel strip, 
immersed into the thermostated chamber filled with perfluorodecalin, can be studied for hours even days without any risk of drying. In the following, all the anisotropic scattering experiments have been carried out during at least 1 hour for each sample in a given deformation state. For all the analyses, the efficiency of the detector cell was normalized by the intensity delivered by a pure water cell of 1-mm thickness and absolute measurements of the scattering intensity I(q) $\left(\mathrm{cm}^{-1}\right.$ or $\left.10^{-8} \AA^{-1}\right)$ were obtained from the direct determination of the incident neutron flux and the cell solid angle. Finally, the coherent scattering intensity of the gel was obtained after subtracting the contribution of the solvent used, as well as perfluorodecalin for samples studied in the oil environment.

\section{Mechanics : tensile test}

Mechanical properties of hydrogels were studied on a tensile Instron machine (model 5565) with a $10 \mathrm{~N}$ load cell (relative uncertainty of $0.16 \%$ in the range from 0 to $0.1 \mathrm{~N}$ ) and a video extensometer (relative uncertainty of $0.11 \%$ at full scale). The typical size of hydrogel samples was $L_{0}=50 \mathrm{~mm}$ for the gauge length, $w_{0}=5 \mathrm{~mm}$ for the width and $t_{0}=2 \mathrm{~mm}$ for the thickness. These gel samples were placed in home-made grips and marked by two white dots in order to follow the local displacement with the video extensometer. The tests were carried out at a constant strain rate: typically $0.06 \mathrm{~s}^{-1}$ which corresponds to an initial velocity of 3 $\mathrm{mm} \cdot \mathrm{s}^{-1}$. The measurements were performed at room temperature and the reproducibility was checked, performing at least three tests per sample formulation. 


\section{RESULTS AND DISCUSSION}

\section{PDMA/silica interactions: adsorption and swelling behavior}

The specific adsorption of poly( $N$-alkylacrylamide) with silica surfaces is a well-known phenomenon that is currently used for example in capillary electrophoresis to reduce or suppress the electroosmotic flow (dynamic coating of capillary) ${ }^{[21-23]}$. It can be noticed that contrary to polyacrylamide that has almost no affinity for silica ${ }^{[24]}$ (the adsorbed amount being very low), the stronger adsorption of $N$-alkyl derivatives such as PDMA originates from the coupling between carbonyl/silanol hydrogen bonding and hydrophobic interactions between methyl groups and silica surface ${ }^{[25-26]}$.

From adsorption experiments, carried out in a previous study at $\mathrm{pH} 8$ between various watersoluble polymers (PNIPAM, PDMA and PEO) and Ludox TM-50 ${ }^{[14]}$, it was shown that PDMA chains readily interact with silica particles; the saturation of silica surfaces being observed at $\Gamma_{\max } \cong 1 \mathrm{mg} \cdot \mathrm{m}^{-2}$. In the case of Ludox TM50 particles, characterized by a specific area $\mathrm{S}_{\text {spe }} \cong 90 \mathrm{~m}^{2} / \mathrm{g}$, the plateau value roughly corresponds to a weight ratio between silica and PDMA: $(S P)_{\Gamma_{\max }} \cong 11$. If we assume a similar plateau value at $\mathrm{pH} 9$, with nevertheless weaker interactions between PDMA and silica, we can consider that all nanohybrid hydrogels investigated in this work, prepared with $\mathrm{S} / \mathrm{P} \leq 5$, contain a large excess of non-adsorbed polymer chains that can readily self-exchange with adsorbed ones.

As mentioned in the experimental part, when embedded inside the PDMA network, the silica nanoparticles remain trapped even after several weeks of swelling experiments in aqueous media. Moreover, the specific interactions taking place between the polymer matrix and the filler introduce geometrical constrains in comparison with the unfilled system. This attractive contribution, which increases with the amount of filler, was shown to be responsible for the continuous decrease of the gel swelling at equilibrium ${ }^{[20]}$. This behaviour, that has been 
described with analytical model based on mechanics of continuous media, is all the more pronounced since the degree of chemical cross-linking is low.

\section{Mechanical reinforcement}

The large strain behavior of hybrid hydrogels was investigated by uniaxial tensile tests for the three series of gels (with $\mathrm{x}$ varying from 0 to 5 and $\mathrm{z}$ from 0 to $1 \mathrm{~mol} \%$ ).

As shown in Table 1, the introduction of silica filler inside the PDMA network greatly enhances the mechanical properties of hydrogels, not only increasing the Young modulus, but also the nominal strain at failure. This general behavior of silica/PDMA hydrogels is a remarkable result in the field of nanocomposite materials and especially in NC gels as generally stiffness and deformability are usually antagonistic properties ${ }^{[27-29]}$. A very large range of mechanical properties can be obtained by tuning either the level of chemical crosslinks (from R0 to R1) or the amount of added silica particles (from SP0 to SP5).

Table 1: Influence of silica particles on Young modulus $(\mathrm{E}, \mathrm{kPa})$ and elongation at break $\left(\varepsilon_{\mathrm{R}}\right)$ for hybrid hydrogels SPx_PW0.14_Rz prepared with different degree of cross-linking.

\begin{tabular}{|l|c|c|c|c|c|c|c|c|c|c|}
\hline & \multicolumn{2}{|c|}{ SP0 } & \multicolumn{2}{c|}{ SP1 } & \multicolumn{2}{c|}{ SP2 } & \multicolumn{2}{c|}{ SP3.5 } & \multicolumn{2}{c|}{ SP5 } \\
\hline & $\mathrm{E}$ & $\varepsilon_{\mathrm{R}}$ & $\mathrm{E}$ & $\varepsilon_{\mathrm{R}}$ & $\mathrm{E}$ & $\varepsilon_{\mathrm{R}}$ & $\mathrm{E}$ & $\varepsilon_{\mathrm{R}}$ & $\mathrm{E}$ & $\varepsilon_{\mathrm{R}}$ \\
\hline R0 & 1 & $>10$ & 2.9 & $>10$ & 6.5 & $>10$ & 15 & $>10$ & 21 & $>10$ \\
\hline R0.1 & 10.5 & 1.5 & 13.8 & 3.5 & 19.8 & 4.1 & 35 & 4.7 & 93 & 6.0 \\
\hline R1 & 44 & 0.25 & 68 & 0.47 & 118 & 0.70 & 160 & 0.67 & 235 & 1.03 \\
\hline
\end{tabular}

The general picture is that both stiffness and strain at break are improved with increasing amount of silica particles (physical cross-links), whatever is the degree of covalent crosslinks. The adhesive contribution of silica/PDMA interactions to the Young modulus $\left(\mathrm{E}_{\mathrm{Si}}\right)$ can be assessed by subtracting the Guth and Gold modulus $\left(\mathrm{E}_{\mathrm{GG}}\right)$ from the initial modulus $(\mathrm{E})$ obtained at very low deformation:

$$
E_{S i}=E-E_{G G}\{1\}
$$

where $\quad E_{G G}=E_{0}\left(1+2.5 \phi_{f}+14.1 \phi_{f}^{2}\right)$ 
with $E_{G G}$ the Guth and Gold modulus that only considers the hydrodynamic contribution of hard spherical particles (volume fraction $\phi_{f}$ ) embedded into an incompressible matrix (modulus $E_{0}$ ) in the absence of specific interaction between the filler and the matrix. The representation of $E_{S i}$ versus the volume fraction of silica particles $\left(\phi_{f}\right)$ plotted in Figure 1a clearly points out the adhesive behavior of the PDMA matrix with silica surfaces that is schematically pictured in the same Figure 1. Indeed, $E_{S i}$ rapidly increases with increasing concentrations of filler due to the formation of a transient network between PDMA and silica.

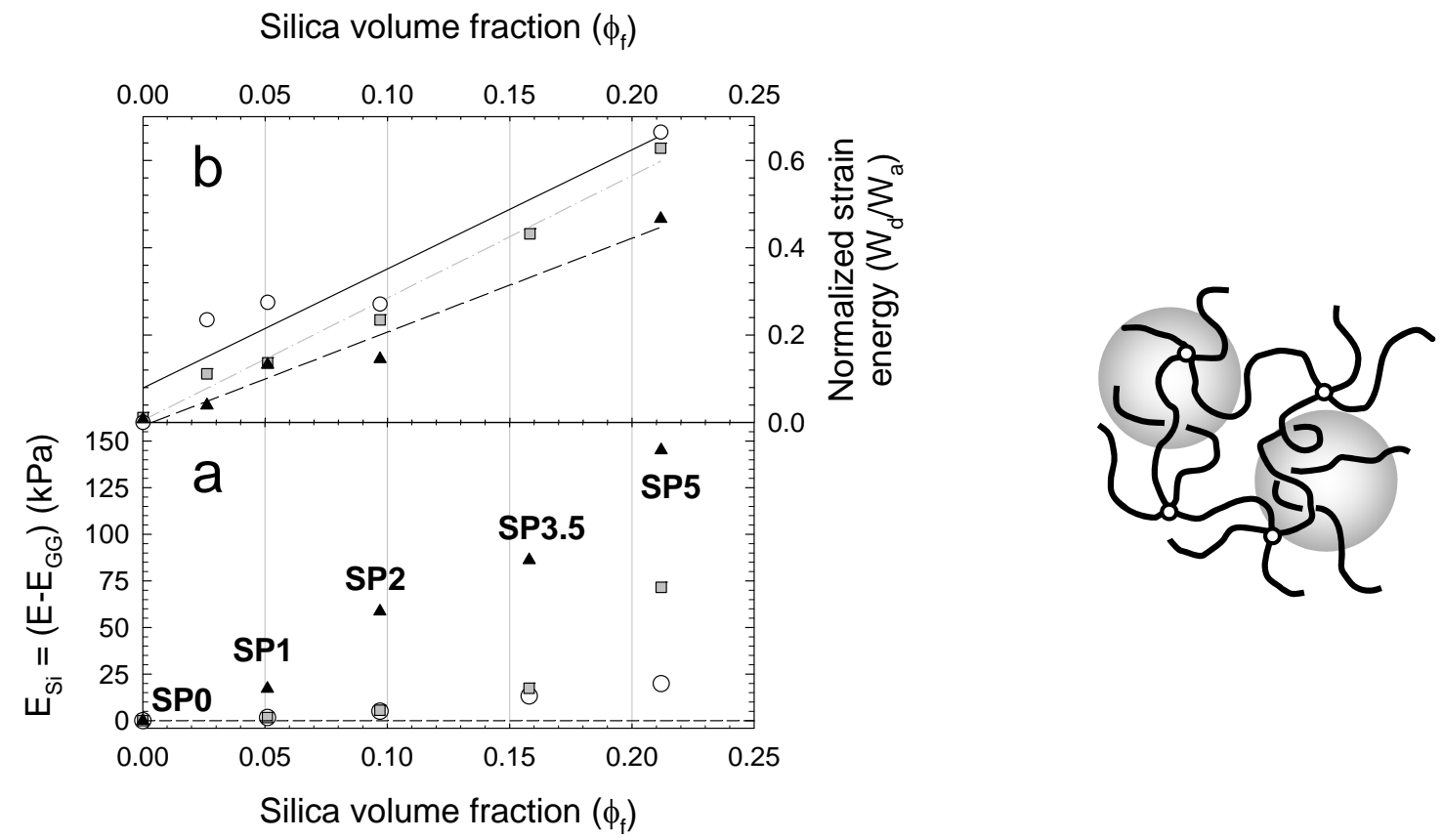

Figure 1: (a) Contribution of silica particles to the initial modulus $\left(\mathrm{E}_{\mathrm{Si}}\right)$ of "SPx_PW0.14_Rz" hydrogels $\left(\mathrm{d} \varepsilon / \mathrm{dt}=0.06 \mathrm{~s}^{-1}\right)$, with $\mathrm{E}_{\mathrm{Si}}=\mathrm{E}-\mathrm{E}_{\mathrm{GG}} ; \mathrm{E}_{\mathrm{GG}}$ being the Guth and Gold modulus taking into account the hydrodynamic contribution of the particles.

(b) Normalized strain energy $W_{d} / W_{a}$ for SPx_PW0.14_Rz series at $0.06 \mathrm{~s}^{-1} ; W_{a}$ being the applied strain energy. The maximal strain was varied from $50 \%, 30 \%$ and $10 \%$ for R0 (O), R0.1 ( $\square)$ and R1 ( $\mathbf{\Delta})$, respectively.

Obviously, $E_{S i}$ increases not only with the amount of silica connectors but also with the level of chemical cross-links. In order to understand this interdependence between physical and chemical cross-links, we have to consider that in nanohybrid hydrogels transient elastic chains are created as soon as PDMA segments interact with silica surfaces and the critical parameter will be the size of the new elastic chains. Indeed, while the average number of monomer units 
between two covalent cross-links obtained from mechanical data varies from 120 to 1200 and more, for R1, R0.1 and R0 respectively, the average degree of polymerization of transient elastic chains created in the surrounding of silica particles should be much lower. In this case, we expect an increasing non-Gaussian behavior with decreasing size of the chains (or increasing degree of cross-linking) with a strong coupling between covalent and physical bonds. A similar mechanism, with the formation of trapped entanglements exhibiting short chain Langevin statistics in the layer of the filler, was proposed by Sternstein and Zhu as major contributor to the stiffening of nanofilled polymer melts ${ }^{[30]}$.

By analogy, the mechanical behaviour of hybrid hydrogels can be conceptually described on the basis of a Zener model coupling in parallel a spring element, taking into account the elasticity of the organic cross-linked network, and Maxwell elements that consider the time dependence of the physical contribution of PDMA/silica to the Young modulus. The superposition of these contributions highlights the possibility to readily tune the hydrogel stiffness playing with the density of cross-links, either physical (SP ratio) or covalent (R). As physical interactions are reversible in nature, with a characteristic time distribution, the adsorption/desorption process of PDMA chains on silica surfaces can be probed by applying stress relaxation experiments on longer timescales or cycling tests at different velocities. This was studied in details with the R0.1 series by changing the strain rate of loading/unloading cycles $^{[19]}$. It was shown that the specific interactions between PDMA chains and silica surfaces are very efficient to reinforce the mechanical properties of the covalent network on short timescales, but not on longer times for which the reinforcement mainly vanishes owing to the reversibility of adsorption. The dynamic behavior of the polymer binding underlines the absence of effective covalent bonds between PDMA and silica particles as well as the absence of an immobilized glassy polymer layer at the silica surface as it has been reported with similar nanocomposite hydrogels ${ }^{[31]}$. The dynamic character of physical interactions between 
PDMA chains and silica particles is also responsible of the self-adhesive properties of hybrid hydrogels. A snapshot of the dissipation process in hybrid hydrogels at a given strain rate, here $0.06 \mathrm{~s}^{-1}$, can be obtained by plotting the dissipated strain energy $\left(W_{d}\right)$ normalized by the strain applied energy ( $W_{a}$; the area under the loading curve) versus the silica volume fraction. The data plotted in Figure 1b show that the normalized strain energy scales almost linearly with the fraction of silica particles and consequently with the amount of adsorbed PDMA chains, given that the hybrid hydrogels were prepared well above the saturation of silica surfaces. On the other hand, the level of covalent cross-linking does not seem to have a significant impact on the dissipation process.

All these macroscopic analyses bring relevant information that clearly emphasize the key-role of specific interactions taking place between silica nanoparticles and the PDMA network on the mechanical reinforcement of organic hydrogels. At this stage, it is important to investigate how the silica nanoparticles, acting as inorganic stickers, self-organize and interact inside the organic matrix before polymerization and after formation of the gel, at rest or under deformation.

\section{Characterization of silica dispersion}

SANS is a very interesting technique to investigate the local structure of complex materials such as hybrid hydrogels. For binary systems like silica suspension, where centrosymetric spherical particles (the filler: index f) are dispersed in a homogeneous medium (the solvent: index s), the scattering intensity can be described by the following relation:

$$
I(q)=\frac{N_{f} V_{f}}{V} \phi_{s}(\Delta \rho)^{2} P(q) S(q)
$$

with $q$ the scattering vector, $N_{f}$ the number of filler particles of average volume $V_{f}=4 \pi R_{f}^{3} / 3$ and radius $R_{f}, V$ the total scattering volume, $\phi_{s}=1-\phi_{f}$ the volume fraction of 
the solvent ( $\phi_{f}=N_{f} V_{f} / V$; being the volume fraction of filler), $\Delta \rho=\rho_{f}-\rho_{s}$ the difference of scattering length densities between the filler and the solvent, $P(q)$ the form factor of the particles and $S(q)$ their structure factor.

A convenient way to compare scattering curves from different formulations is to divide $\mathrm{I}(\mathrm{q})$ in $\{$ Eq.3 $\}$ by $\phi_{f} \phi_{s}(\Delta \rho)^{2}$ or by using the experimental value of the invariant given by:

$$
Q_{\exp }=\int_{0}^{\infty} q^{2} I(q) d q
$$

Indeed, for an incompressible biphasic system, the invariant is in theory a constant that only depends on the volume fraction and the contrast between the two phases:

$$
Q_{t h}=2 \pi^{2}(\Delta \rho)^{2} \phi_{f} \phi_{s}
$$

In the case of particles with sharp interfaces, the scattering intensity, as well as $q^{2} I(q)$, rapidly decreases with q following the Porod's law $\left(I(q) \approx q^{-4}\right)$ at high values of $\mathrm{q}$. Thus, if the Porod regime is reached over the experimental chosen q-range, the integral in $\{$ Eq. 4$\}$ can be calculated. We will use it in the following relation to describe the scattering data:

$$
I_{c o r}(q)=2 \pi^{2} \frac{I(q)}{Q_{\exp }}=P(q) S(q)
$$

The correction of the scattering intensity by the experimental value of the invariant is indeed a proper way to minimize experimental uncertainties related to concentration and contrast from one sample to another. $I_{c o r}(q)$ enables to focus on the relevant scattering functions in the system: (i) the form factor $\mathrm{P}(\mathrm{q})$ that accounts for the interference of neutrons scattered from different parts of the given object and gives geometrical information about its size and shape and (ii) the structure factor $\mathrm{S}(\mathrm{q})$ that account from the interference of neutrons scattered from different objects and provides details about their correlation. S(q) is the Fourier transform of the correlation function of the mass centre of the particles. 
For Ludox-TM50, which are polydisperse spherical particles, $\mathrm{P}(\mathrm{q})$ can be described by the following expression:

$$
P(q)=\int_{-5 \sigma}^{+5 \sigma} \frac{4 \pi r^{3}}{3}[F(q, r)]^{2} \omega(r) d r \quad\{7\}
$$

with $F(q, r)=3 \frac{\sin (q r)-q r \cos (q r)}{(q r)^{3}} \quad\{8\} \quad$ and $\omega(r)=\frac{1}{\sqrt{2 \pi} \sigma} \exp \left(-\frac{(r-R)^{2}}{2 \sigma^{2}}\right)$

where $\omega(r)$ is the Gaussian size distribution function with $\sigma$ the standard deviation.

In dilute condition, when interactions between particles are negligible, $S(q)=1$ over the whole range of q and $I_{c o r}(q)$ is equal to the form factor. With a dilute dispersion of Ludox TM50 ( $1 \mathrm{wt} \%)$, the best fitting obtained from the polydisperse sphere model gives a particle radius $R_{f}=132 \AA$ with a standard deviation $\sigma=15 \AA$ (see Figure S2 in supporting information).

At higher silica concentrations, typically in the range where hybrid hydrogels are prepared, colloidal interactions are important and the structure factor $\mathrm{S}(\mathrm{q})$ is no longer equal to 1 . When the particles have a liquid-like order in solution, the structure factor has a marked correlation peak $q_{\max }$ at intermediate $\mathrm{q}$ which characterizes the average distance between the particles and a low intensity at very low q, i.e. a weak osmotic compressibility $(\chi=S(0))$. This situation is clearly depicted in Figure 2 that shows the scattering pattern of a concentrated suspension of Ludox TM50 $\left(\mathrm{C}=20 \mathrm{wt} \%\right.$ or $\left.\phi_{f}=0.099\right)$ with a strong correlation peak at $q_{\max }=0.015 \AA^{-1}$. Please note that the q-position of this maximum can be shifted from that of $\mathrm{S}(\mathrm{q})$ by the decreasing function of $\mathrm{P}(\mathrm{q}) . \mathrm{S}(\mathrm{q})$ can be deduced by dividing $I_{\text {cor }}(q)$ by $\mathrm{P}(\mathrm{q})$, assuming that the form factor previously determined in dilute conditions corresponds to the one of isolated particles. Such treatment is presented in Figure 2 (inset). It shows clear-cut feature in the qrange of interest of the structure factor with: (i) a clear correlation peak around $q_{\max }=0.017$ $\AA^{-1}$ and (ii) a sharp decrease of $\mathrm{S}(\mathrm{q})$ below 1 at low $q$, pointing out the reduction of the 
osmotic compressibility of the suspension with increasing concentration which demonstrates that the interactions between silica particles are repulsive on average.

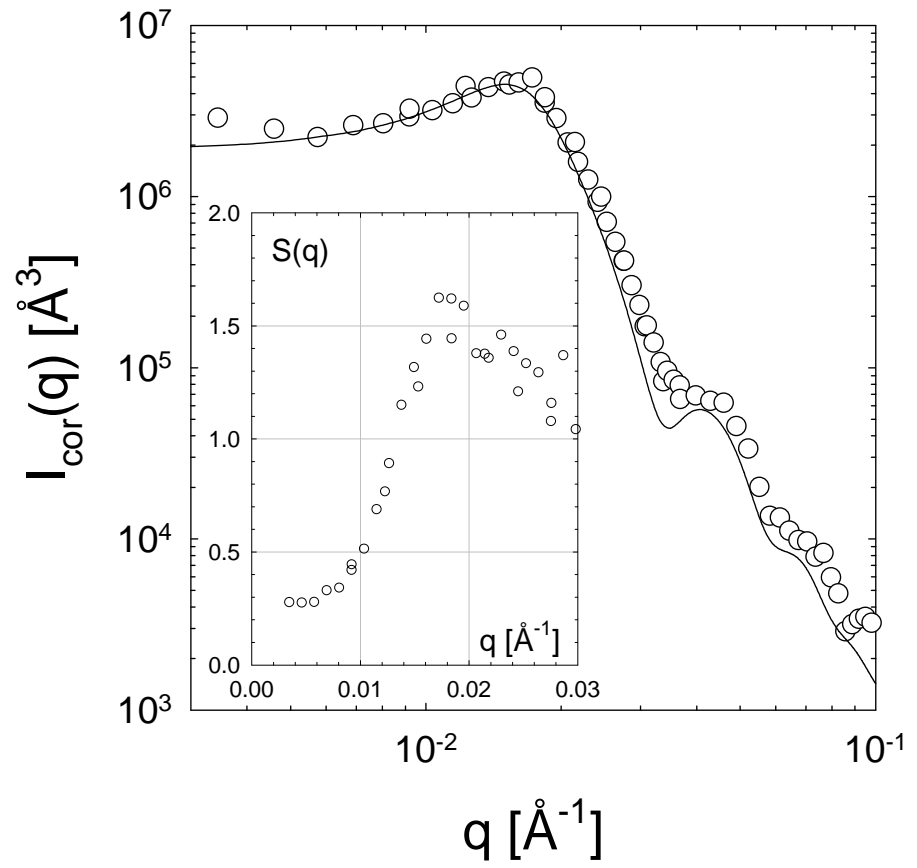

Figure 2. Double logarithmic plots of the reduced scattering intensity $(\mathrm{O})$ obtained from a concentrated dispersion of silica particles $\left(C=20 \mathrm{wt} \%\right.$ or $\left.\phi_{f}=0.099\right)$. The corresponding fit based on a polydisperse sphere model with hard sphere repulsions is indicated in solid line. Inset: Representation of the experimental structure factor obtained by dividing the reduced scattering intensity of concentrated silica suspension $\left(\phi_{f}=0.099\right)$ by the reduced scattering intensity of dilute silica suspension $\left(\phi_{f}=0.0044\right)$.

In order to model the whole scattering behavior of silica dispersions with a minimum number of adjustable parameters, we have assumed in the following that the interactions between particles can be described by a hard sphere potential and their radius $R_{f}$ renormalized by an effective radius $R_{H S}$, the volume fraction becoming $\phi_{H S}=\phi_{f}\left(R_{H S} / R_{f}\right)^{3}$. The structure factor S(q) can then be calculated from the Percus-Yevick approximation (see details in supporting information). Since $\phi_{f}$ and solvent composition are known from synthesis, the experimental scattering intensities can be fitted in absolute units according to equation $\{6\}$ by using a simple set of unequivocal variables: $R_{f}, \sigma$ and $R_{H S}$. Such modeling is presented in Figure 2 for the Ludox suspension $\left(\mathrm{C}=20 \mathrm{wt} \%\right.$ or $\left.\phi_{f}=0.099\right)$. In that case, the fitting procedure gives i) $R_{f}$ $=134 \AA$, similar to the value obtained in dilute conditions, ii) an average polydispersity 
$\sigma(\mathrm{R}) / \mathrm{R}$ of about $10 \%$ in good agreement with the literature data ${ }^{[32]}$ and iii) a hard sphere radius of $175 \AA$. The difference between the effective hard sphere radius and the physical one enables to probe the range of the weak electrostatic repulsions that exist between silica nanoparticles which is of the order of the Debye length; i.e. 20 to $30 \AA$ in our conditions. Prior to fitting, a raw estimate of these two parameters $R_{f}$ and $R_{H S}$ can be first obtained from the features of the scattering curve, assuming that the correlation peak is related to the most probable distance between particles $\left(q_{\max }=0.0172 \AA^{-1}\right.$ and $\left.R_{H S}=182 \AA\right)$, followed by the minima related to the oscillations of the form factor, is $q_{\min } R=4.5$; i.e. $R_{f}=128 \AA$ for $\mathrm{q}_{\min }=0.035 \AA^{-1}$.

\section{Characterization of silica dispersion in hybrid hydrogels}

As far as hybrid hydrogels are concerned, we have to consider the study of a ternary system composed of cross-linked polymer (p), filler (f) and solvent (s). This situation cannot longer be described by the previous general structure factor; the three components must be taken into account as:

$$
I(q)=\left(\rho_{p}-\rho_{s}\right)^{2} \bar{S}_{p p}(q)+\left(\rho_{f}-\rho_{s}\right)^{2} \bar{S}_{f f}(q)+2\left(\rho_{p}-\rho_{s}\right)\left(\rho_{f}-\rho_{s}\right) \bar{S}_{p f}(q)
$$

with $\rho_{i}$ the scattering length density of each component and $\bar{S}_{i j}(q)$ the partial scattering functions: $\bar{S}_{p p}(q)$ and $\bar{S}_{f f}(q)$ are respectively the self-correlations of the polymer and the silica filler and $\bar{S}_{p f}(q)$ represents the cross-correlation between the polymer and the filler ${ }^{[33]}$. In the case of solvent $\mathrm{p}$, where both polymer and solvent mixture have the same scattering length density, equation $\{10\}$ is reduced to equation $\{3\}$ and all the procedure previously described with silica suspension can be readily applied to hybrid hydrogels. As shown in Figure 3, where the scattering behavior of hybrid networks with various amount of crosslinkers and initial silica suspension are compared at the same filler concentration $\left(\phi_{\mathrm{f}}=\right.$ 
0.099), we get a very good superposition of the curves in the high q-range which is mainly dominated by the form factor of silica particles $(\mathrm{S}(\mathrm{q}) \cong 1)$.

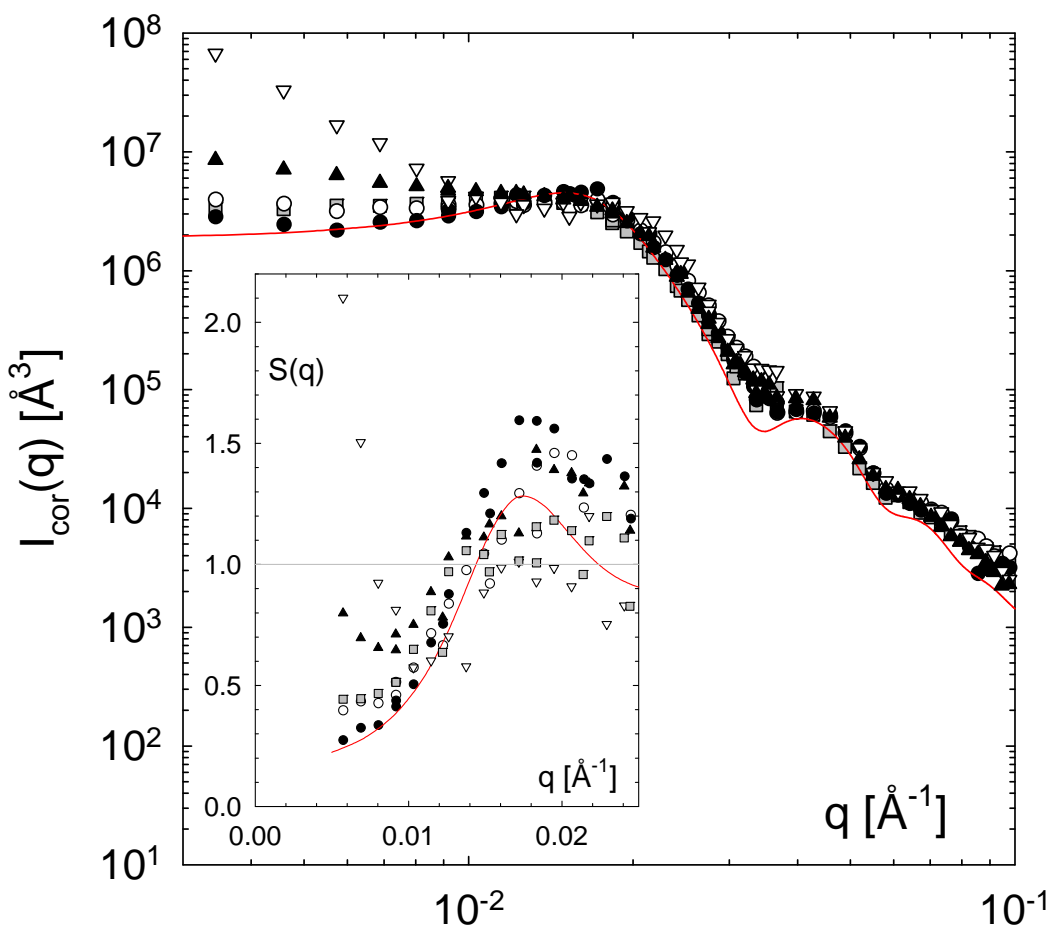

Figure 3: Double logarithmic plots of the reduced scattering intensity of PDMA/silica hydrogels (SP2_PW0.14_Rz) prepared in solvent p (silica scattering) with different amount of cross-linker: R0 (O); R0.1 ( $\square)$; R1 ( $\mathbf{\Delta})$ and R5 ( $\nabla)$. By comparison, the scattered intensity of silica particles without PDMA but at the same volume fraction $\left(\phi_{\mathrm{f}}=0.099\right)$ and in the same solvent conditions is shown (O) with the fit (solid line) obtained from the hard sphere model. (Inset) Representation of the experimental structure factor obtained by dividing the reduced scattering intensity of hybrid formulations by the reduced scattering intensity of dilute silica suspension $\left(\phi_{\mathrm{f}}=0.0044\right)$.

The main difference arises at low q where the scattering intensity increases when silica beads are embedded into the PDMA network with increasing level of cross-linking. This difference originates from the structure factors of the particles which are shown in the inset of Figure 3 in the q-range of interest. As the distribution of silica particles in the polymer network becomes rapidly locked, as soon as the system reaches the gelation threshold, we can reasonably assume that the structure factor of silica particles mirrors the first stage of the polymerization. By comparison with the silica suspension, the polymerization of DMA without ( $\mathrm{z}=0 \mathrm{~mol} \%)$ or with a low level of cross-linker ( $\mathrm{z}=0.1 \mathrm{~mol} \%)$, only slightly increases 
the osmotic compressibility of the system which remains repulsive in nature, but of lower intensity. In this case it is possible to fit the scattering by the hard sphere model described for pure silica suspensions. We observed a decrease of the values of $\mathrm{R}_{\mathrm{HS}}$ in the hybrid suspension (155 $\AA$ for both $\mathrm{z}=0 \mathrm{~mol} \%$ and $\mathrm{z}=0.1 \mathrm{~mol} \%$ ) from the one of the pure silica suspension (175 $\AA$ ), showing that the range of such repulsions has decreased. At higher level of cross-linking $(\mathrm{z}=1$ and $5 \mathrm{~mol} \%$ ) S(q) goes upward at low $\mathrm{q}$ and even becomes higher than 1 for the highest level of cross-linker. In that case the potential between particles becomes clearly attractive and tends towards aggregation. The destabilization of silica particles in the presence of polymers can be understood on the basis of enthalpic or entropic contributions.

Enthalpic contribution could prevail when there is a strong attractive potential between polymer and particles like between negatively charged silica at $\mathrm{pH} 9$ and cationic polymers or as it was observed by Shibayama and co-workers ${ }^{[33]}$ during the polymerization of $\mathrm{N}$ isopropylacrylamide at low pH (between 3 and 6) in the presence of silica particles. Entropic effects are generally related to the formation of a depletion layer of polymer chains near the surface of the particles. In the case of PDMA/silica, characterized by attractive interactions, a layer of cross-linked PDMA is rapidly formed (adsorbed) at the silica interface during the first stage of the copolymerization. This layer provides an additional steric stabilization of the particles, but it is also known that sterically stabilized particles can undergo weak flocculation when suspended in a polymer solution of the same composition ${ }^{[34,35]}$. If we assume that, at the beginning of the reaction the first polymerized chains are adsorbed on the silica particles, this polymerization/adsorption mechanism will initially drive a concentration difference between the polymer layer and the bulk. If the concentration of the polymer layer is controlled by the degree of cross-linking during the first stage of the polymerization it follows that the depletion mechanism should increase with the degree of cross-linking with a higher tendency towards aggregation as it was shown in Figure 3. In these conditions, the bridging forces 
induced by PDMA chains and then the level of heterogeneity of the hybrid network are expected to increase with parameters such as binding energy or cross-linking density. The aggregation mechanism is particularly favourable at high degree of cross-linking, typically for $\mathrm{z}$ above $2 \mathrm{~mol} \%$, where syneresis has been observed during the synthesis.

With increasing fraction of filler inside the network, we observe in Figure 4 a sharp decrease of I(q) at low q due to large evolution of the structure factor while the scattering pattern is constant in the high q-range where one probes only $\mathrm{P}(\mathrm{q})$. In that case, a correlation peak is readily observed from $\phi_{\mathrm{f}} \cong 0.1(\mathrm{SP} 2)$. The experimental data can be reasonably fitted with the hard sphere model (see supporting information Figure S3), showing a slight decrease of $R_{H S}$ with decreasing fraction of filler (Table 2), except for the lowest filler concentration (SP0.5) which is dominated by attractive interactions and cannot be described by the hard sphere potential.

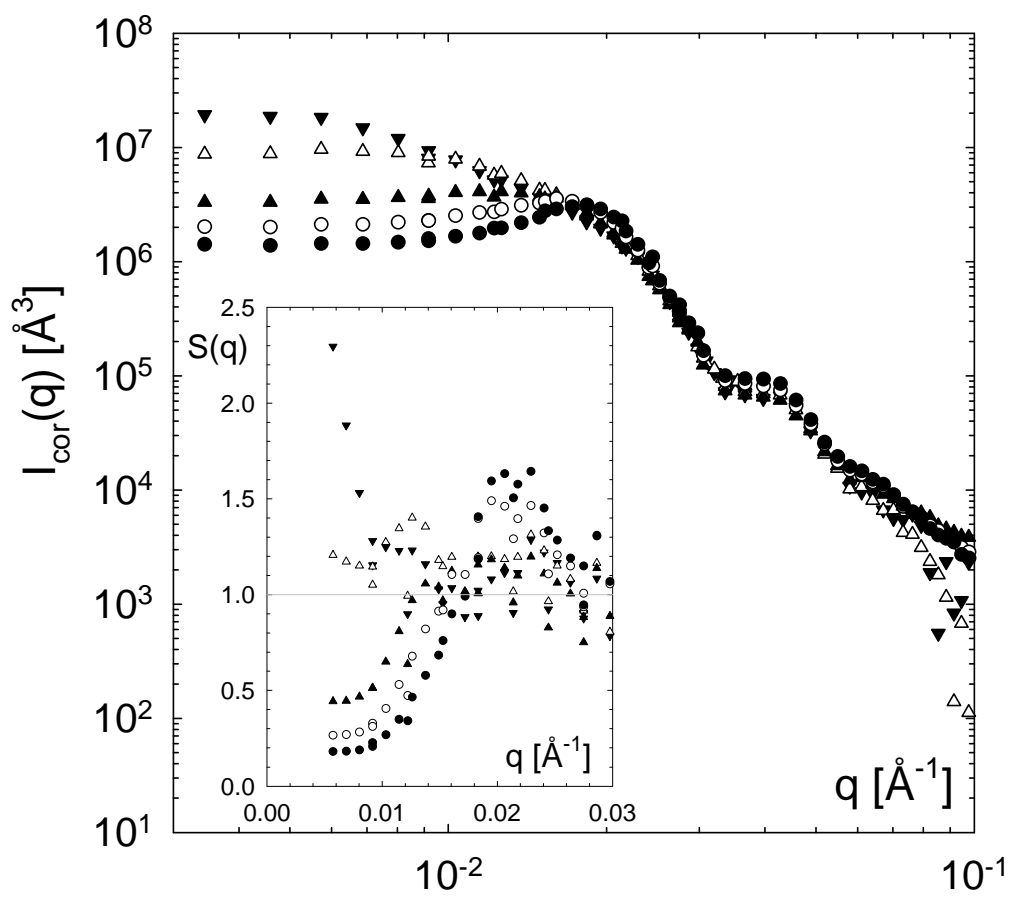

Figure 4: Double logarithmic plots of the normalized scattered intensity of PDMA/silica hydrogels (SPx_PW0.14_R0.1) prepared in solvent $\mathrm{p}$ (silica scattering) with different amount of silica: SP0.5 $(\boldsymbol{\nabla}), \operatorname{SP} 1(\triangle)$, SP2 $(\boldsymbol{\Delta}), \operatorname{SP} 3.5(O)$ and SP4.8 (O). Inset: Experimental structure factor obtained by dividing the reduced scattering intensity of hybrid formulations by the reduced scattering intensity of dilute silica suspension $\left(\phi_{\mathrm{f}}=0.0044\right)$. 
The plot of the $S(q)$ in Figure 4 emphasizes the variation of the osmotic compressibility $(\mathrm{S}(0))$ with the concentration of filler. Whereas the potential is clearly repulsive for volume fraction equal and higher than $\phi_{\mathrm{f}} \cong 0.1$, it progressively turns attractive at lower concentration and especially for the sample $\mathrm{SP} 0.5\left(\phi_{\mathrm{f}} \cong 0.025\right)$. One of the main parameter that is varied during preparation of hybrid hydrogels at various filler concentrations is the dilution of the original dispersion of Ludox-TM50 suspension initially stabilized at $\mathrm{pH}$ 9. While the silica suspension is diluted only 1.4 times to formulate the sample SP5, the dilution is 14 times for the SP0.5. The decrease of the $\mathrm{pH}$ upon dilution enhances the interactions between PDMA and silica surfaces $(\mathrm{SiOH})$, which could explain the variation of $\mathrm{S}(\mathrm{q})$ with the filler content. From this set of experiments, neutron scattering demonstrates the possibility to get well dispersed silica particles inside PDMA networks as long as they were prepared with a reasonable level of cross-linker.

\begin{tabular}{|l|c|c|c|c|}
\hline \multicolumn{5}{|c|}{$\begin{array}{c}\text { Table 2. Fitting parameters related to silica scattering obtained from } \\
\text { hybrid hydrogels prepared in solvent } \mathrm{p}\left(\phi_{\mathrm{D} 2 \mathrm{O}}=0.21 ; \phi_{\mathrm{H} 2 \mathrm{O}}=0.79\right) .\end{array}$} \\
\hline Formulation & $\phi_{\mathrm{f}}$ & $\mathrm{R}_{\mathrm{f}}(\AA)$ & $\sigma(\AA)$ & $\mathrm{R}_{\mathrm{HS}}(\AA)$ \\
\hline SP0.5_PW0.14_R0.1 & 0.027 & 135 & 15 & --- \\
\hline SP1_PW0.14_R0.1 & 0.052 & 135 & 15 & 135 \\
\hline SP2_PW0.14_R0.1 & 0.099 & 135 & 15 & 155 \\
\hline SP3.5_PW0.14_R0.1 & 0.161 & 135 & 15 & 148 \\
\hline SP4.8_PW0.14_R0.1 & 0.206 & 135 & 15 & 148 \\
\hline
\end{tabular}

For these systems, we assume that there is an attractive potential between silica and PDMA, mainly driven through hydrogen bonds and hydrophobicity as it has been reported for polymer coating on fused silica capillaries ${ }^{[25,36]}$. Nevertheless, this attractive potential is not too high at $\mathrm{pH} 9$ to induce any strong aggregation.

\section{Looking for the corona}

The behaviour of hybrid hydrogels appears to be driven by a PDMA layer adsorbed at the silica interface, that has been experimentally evidenced from adsorption isotherm 
measurements ${ }^{[16]}$. We try to get here a better characterization of such a layer by neutron scattering, especially about its concentration.

Working in solvent $\mathrm{f}$, the filler and the solvent have the same scattering length densities $\left(\rho_{\mathrm{s}}=\rho_{\mathrm{f}}\right)$ and the situation is now similar to a PDMA hydrogel with solvent holes replacing the silica nanoparticles. This case is nevertheless different from the previous one as we assume that PDMA chains interact with silica surfaces and we have to consider a 3-phase model from the neutrons point of view with: 1) the filler (index f), 2) the adsorbed polymer layer at the interface (index $l$ ) and 3) the PDMA matrix which does not interact directly with the particles (index $m$ ). In order to take into account this situation we have used the form factor of a coreshell particle that sums the scattering between the hard core and the corona (adsorbed polymer layer) and between the corona and the surrounding polymer matrix. Its scattering amplitude is given by ${ }^{[33]}$ :

$$
\begin{aligned}
A(q) & =V_{l}\left(\rho_{l}-\rho_{m}\right) F\left(q, R_{l}\right)+V_{f}\left(\rho_{f}-\rho_{l}\right) F\left(q, R_{f}\right)\{11\} \\
\text { or } \quad A(q) & =\left(\rho_{p}-\rho_{s}\right)\left\{\left(\phi_{l}-\phi_{m}\right) V_{l} F\left(q, R_{l}\right)-\phi_{l} V_{f} F\left(q, R_{f}\right)\right\}
\end{aligned}
$$

with $\phi_{1}$ and $\phi_{m}$ the volume fraction of polymer in the layer and out of the layer, $F\left(q, R_{i}\right)$ the scattering amplitude of spheres of radius $\mathrm{R}_{\mathrm{i}}$ defined by $\{\mathrm{Eq} .8\}, V_{i}=4 \pi R_{i}^{3} / 3$ the volume of spheres with $R_{l}=R_{f}+t$ where $\mathrm{t}$ is the thickness of the adsorbed polymer layer.

In order to describe the whole scattering intensity, we have to take into account the structure factor of the particles. As the position of the mass centre of the silica beads coincides with that of the polymer layer, the structure factor $\mathrm{S}(\mathrm{q})$ is similar as the one described in the previous section for the measurements in solvent $\mathrm{p}$. I(q) can be then given as follows:

$$
I(q)=\frac{N_{f}}{V} \phi|A(q)|^{2} S(q) \quad\{13\} \quad \text { with } \quad \phi=1-\left(N_{f} V_{l} / V\right)
$$

In this relation we neglect the scattering from concentration inhomogeneities of the polymer network, as this contribution is very weak in solvent $\mathrm{f}$. 
In order to probe the influence of variations of either the thickness of the polymer layer or its concentration on the scattering profile of core-shell particles, simulations have been carried out using \{Eq.12\} (see Figure S4 in supporting information). These simulations point out dramatic changes of scattering patterns due to i) large variations of the scattering volume $\left(\{A(0)\}^{2}=\left\{V_{l}\left(\rho_{l}-\rho_{m}\right)+V_{f}\left(\rho_{f}-\rho_{l}\right)\right\}^{2}\right)$ as a function of the characteristics of the polymer layer (thickness and concentration) and ii) interferences between the core and the shell that give rise to additional oscillations in the scattering spectrum. Whereas similar patterns are expected from $\{$ Eq.13\} for $\mathrm{I}(\mathrm{q})$ versus $\mathrm{q}$, we do not observe experimentally large modifications of the scattering profiles with contrast variation of the solvent. An example is given in Figure 5 for a given sample SP2_PW0.14_R0.1 prepared in 3 different conditions: $\mathrm{H}_{2} \mathrm{O}$, solvent $\mathrm{p}$ and solvent $\mathrm{f}$.

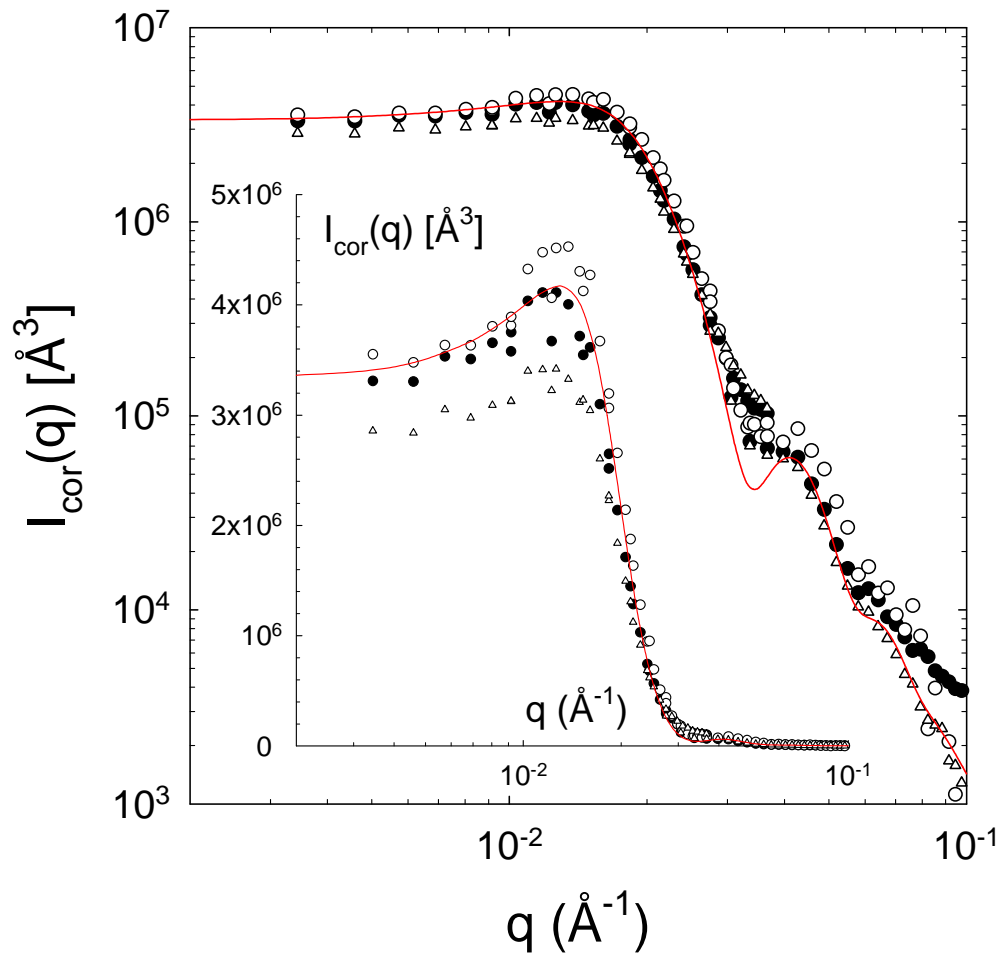

Figure 5: Double logarithmic plots of the normalized scattered intensity of PDMA/silica hydrogels (SP2_PW0.14_R0.1) prepared in various solvents: $(O) \mathrm{p}\left(\phi_{\mathrm{D} 2 \mathrm{O}}=0.21 ; \phi_{\mathrm{H} 2 \mathrm{O}}=0.79\right)$ $(\mathrm{O}), \mathrm{f}\left(\phi_{\mathrm{D} 2 \mathrm{O}}=0.58 ; \phi_{\mathrm{H} 2 \mathrm{O}}=0.42\right)$ and pure $\mathrm{H}_{2} \mathrm{O}(\triangle)$. The corresponding fit based on a polydisperse sphere model with hard sphere repulsions is indicated in solid line. Experimental data and model fitting are also shown under a semi-log form in the inset. 
As we can see, the three plots have qualitatively the same signature. The fit obtained when the polymer is contrast-matched in solvent $\mathrm{p}$ presented in previous section describes thus pretty well the other scattering curves. This behaviour is a general trend that we have observed for all the formulations of the R0.1 series which have been studied in the three different solvents. Although the PDMA chains interact with silica surfaces, these results show that they do not form a concentrated layer at the interface of significant thickness. Indeed, if a concentrated layer is formed, its thickness would be necessary very low and beyond the experimental accuracy to give any noticeable signature on the scattering pattern. By comparison, Shibayama and co-workers, who worked with silica particles embedded into a PNIPAM gel matrix prepared at lower concentration (around 5-6 vol\%) have determined a polymer layer with a thickness of about $30 \AA$ and a volume fraction of 15 to 17 vol\% ${ }^{[33]}$. Even with PNIPAM which is known to strongly interact with silica particles, especially at low $\mathrm{pH}$ as it was the case in their study, the concentration of the polymer layer was finally not very high and of the same order of our gel formulation (12 to 13 vol\%). Thus, if a layer of this magnitude of volume fraction is formed around the silica particles in our case, its average neutron scattering length density would be very close to the one of the solvated PDMA matrix, and the system will behave as a two-phases system from neutron point of view in solvent $\mathrm{f}$, as observed in Figure 5. Similarly, we can also mention the work of Qiu et al ${ }^{[37]}$ who showed that poly(ethylene oxide) chains adsorb onto colloidal silica particles forming a "dense" layer with a PEO volume fraction at the interface increasing from 10 to $12 \mathrm{vol} \%$ with silica concentration increasing from 1 to $20 \mathrm{vol} \%$. Here again we have to take care not to confuse dense layer and immobilized layer that should correspond to much higher concentrations in the case of PDMA. 


\section{Small angle neutron scattering under deformation}

SANS is widely used to analyze the structure of polymer networks under deformation as thermal fluctuations of polymer chains, in a few cases, and frozen inhomogeneities, much more often, give rise to very informative anisotropic patterns ${ }^{[38-42]}$. A first kind of deformation that can be applied on hydrogels is their swelling and deswelling, emphasizing their structural inhomogeneities. The second kind of deformation is the mechanical stretching of hydrogels. The complex spatial distribution of strains and stresses in a deformed nanocomposite material is indeed a crucial problem for understanding the reinforcement mechanisms.

In the framework of this study we mainly investigate the structure of silica particles which are used in this case as local probes (or tracers) during the deformation of the hydrogel. For all the scattering patterns, the direction of the uni-axial deformation is along the horizontal axis. A first set of experiments is shown in Figure 6 with the hybrid hydrogel SP5_PW0.14_R0.1 studied under incremental strain, from $\varepsilon=0$ to $200 \%$.
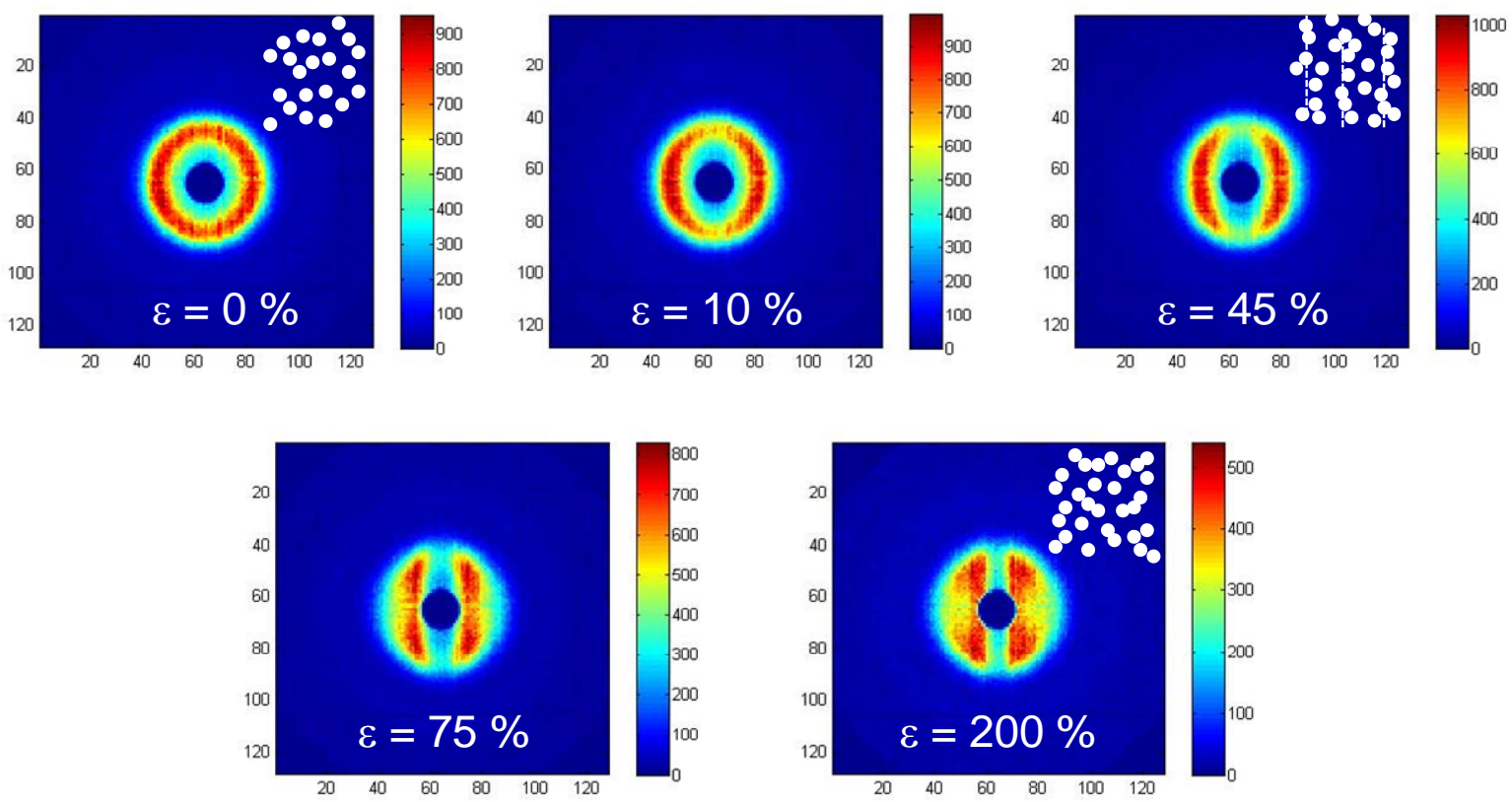

Figure 6: 2D-SANS images of a step-by-step loading of SP5_PW0.14_R0.1 hybrid hydrogel. The white dots in the upper right corner schematically picture the average distance between silica particles in hybrid hydrogels for un-stretched $(\varepsilon=0 \%)$ and stretched materials, before ( $\varepsilon$ $=45 \%)$ and during $(\varepsilon>75 \%)$ shear reorganization. The dotted lines shown at $\varepsilon=45 \%$ highlight the alignment of particles under transverse compression. 
As expected, the unstrained sample displays an isotropic pattern with a circular diffraction ring corresponding to the correlation peak of the structure factor discussed in previous section. This isotropic scattering can also be observed in Figure 7 where the $2 \mathrm{D}$ data have been plotted after sector-averaging, considering three different sectors of $10^{\circ}$ width: the horizontal one $\left(0^{\circ}\right.$ : with $\mathrm{X}$ the stretching direction), the vertical one $\left(90^{\circ}\right.$; $\mathrm{Y}$ being the transverse direction) and the intermediate direction at $45^{\circ}$. At rest, all the scattering curves are superimposed whatever the angular sector is.
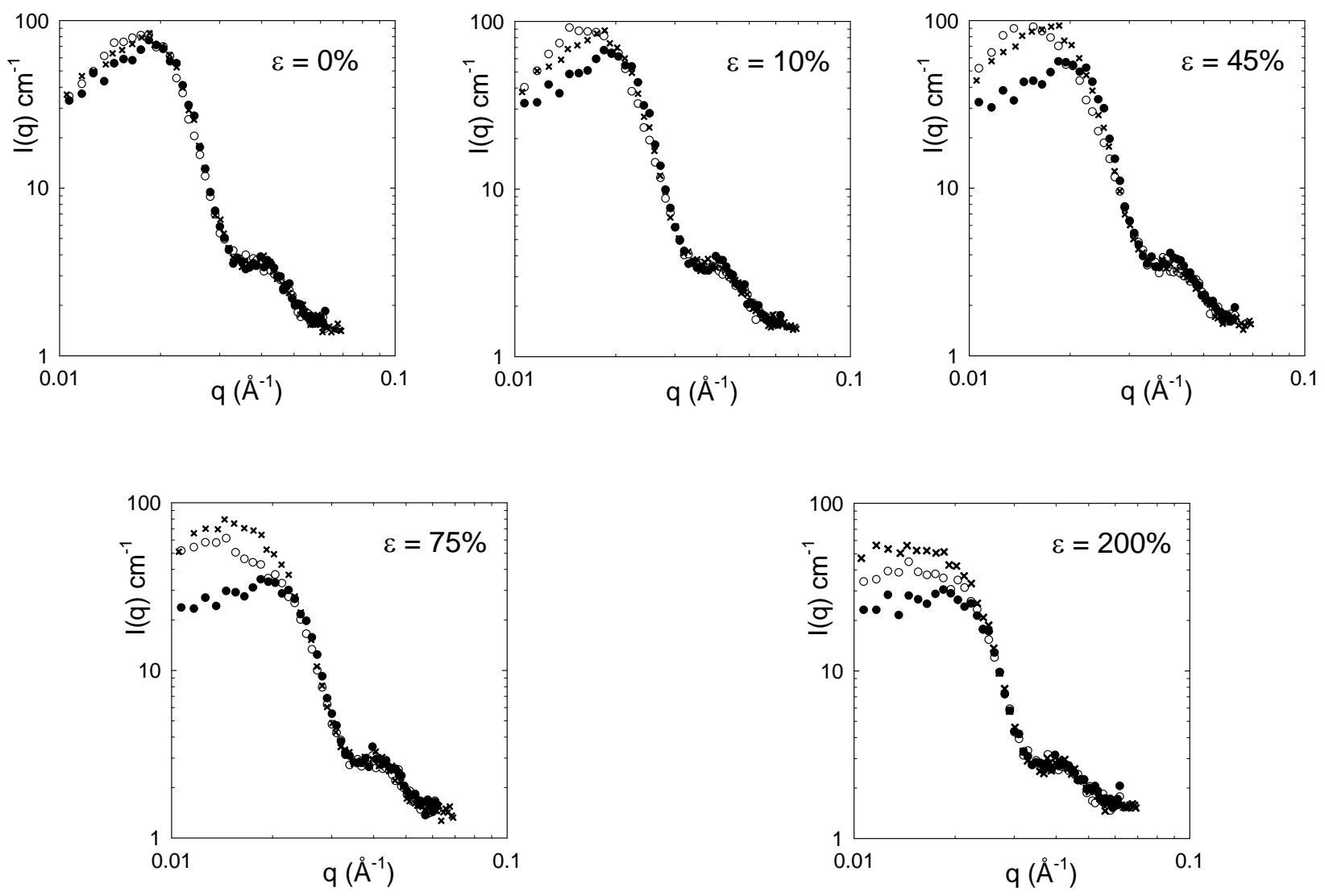

Figure 7: Double logarithmic plot of the scattering intensity of SP5_PW0.14_R0.1 hydrogel studied at different strains: from $0 \%$ to $200 \%$. The data were obtained from 2D-SANS images according to different sector-averaging patterns of $10^{\circ}$ width: $0^{\circ}(\mathrm{O}), 45^{\circ}(\mathrm{X})$ and $90^{\circ}(\mathbf{O})$.

At low deformation, typically for strains of $10 \%$ and $45 \%$, a double wing pattern (or butterfly pattern) appears in Figure 6 as a consequence of an increase of the scattering intensity in the parallel direction and a decrease along the perpendicular one. This situation is 
clearly emphasized in Figure 7 where the intensity increases in the X-direction with a shift of $q_{\max }$ towards low $q$-vectors, while it decreases in the perpendicular direction with a maximum pushed to higher $q$. In the meanwhile, the scattering profile at $45^{\circ}$ remains almost unchanged if we compare $\varepsilon=0,10$ and $45 \%$. It is worth noting that similar patterns have been first reported with silica particles in dry polyacrylate latex films ${ }^{[43]}$ and later on with other hybrid materials like polyisoprene/silica ${ }^{[44]}$ and nanocomposite hydrogels ${ }^{[45-47]}$

These patterns, which originate from the anisotropic space displacement of scatterers, here the silica particles, have also been described by computer simulation by Boué and coworkers $^{[43,48]}$. As schematically sketched in Figure 6 with the white dots depicting the silica particles in the real space, the stretching of the sample along the X-direction, involves an increase of the average distance between silica particles along the same direction while this distance decreases in the perpendicular direction, due to transverse compression. As the particles are brought closer in the Y-direction, their scattering maximum is shifted towards higher $q$ (smaller distances). In the same time, the fluctuations are reduced and the scattering maximum is decreased. Conversely, in the parallel direction, the particles are diluted and the maximum is enhanced at lower $q$. As shown in Figure 7, the modification of the scattering pattern under deformation is mainly observed in the intermediate $q$-range. Indeed, in the high $q$-range, the scattering intensity, proportional to form factor of the silica particles, remains constant as they are non-deformable. When the deformation increases (for $\varepsilon=75$ and $200 \%$ ) the double wing pattern evolves into a set of four maxima which points out some reorganization of the nanoparticles. This morphological change originates from the transverse compression that pushes the particles towards each other. As shown by computer simulation by Rharbi et al. ${ }^{[48]}$, the four-spot pattern implies that the particles, which are pushed towards each other by the deformation of the matrix, may avoid each other by localized shear displacements. Accordingly, a re-densification is expected in the X-direction with a decrease 
of the correlation length along $\mathrm{X}$ and a shift of the correlation peak at higher $q$, thus weakening the diffusion along this axis. This scenario, schematically described in the real space in Figure $6(\varepsilon=200 \%)$, strongly depends on the excluded volume, on the size, polydispersity, concentration and state of aggregation of particles. According to computer simulation $^{[48]}$, it was shown that the number of collisions between particles raises immediately with the deformation in the case of a random hard sphere distribution while it starts at higher deformation for a liquid-like order distribution. A raw estimation of the critical deformation at which collisions between particles become effective, can be calculated assuming a simple cubic lattice of silica particles. For SP5_PW0.14_R0.1 hydrogels, this critical strain is $\varepsilon_{\mathrm{cr}}=83$ $\%$ which has to be compared with the experimentally observed values of $\varepsilon_{\mathrm{cr}}=45-75 \%$ (see Figure 6). Under this consideration, a similar behaviour would be expected for SP2 hydrogels at higher deformation $\left(\varepsilon_{\mathrm{cr}} \cong 200 \%\right)$.

In Figure 7, we have seen that for strained samples $(\varepsilon<75 \%)$, the position of the scattering maximum $\left(q_{\max }\right)$ was shifted towards low $q$ in the stretching direction and high $q$ in the perpendicular direction. Using $q_{\max }$ as a rough estimation of the average distance between particles $\left(d=2 \pi / q_{\max }\right)$, this scaling parameter has been plotted in Figure 8 as a function of strain. In this regime of low deformation, if we assume that the displacement of silica particles is affine with the macroscopic deformation, the opposite of the average distance from centre to centre $\left(q_{\max }\right)$ will decrease by a factor $\varepsilon+1$ in the X-direction and increase with $(\varepsilon+1)^{1 / 2}$ in the perpendicular one. The comparison with affine deformation suggests some deviation that can be attributed to the short-range repulsive inter-particle forces that have been pointed out previously. Moreover, the possibility for polymer chains to exchange reversible contacts between particles when they are close to each other, certainly introduces additional relaxation effects that move the particles away from their affine displacement. 


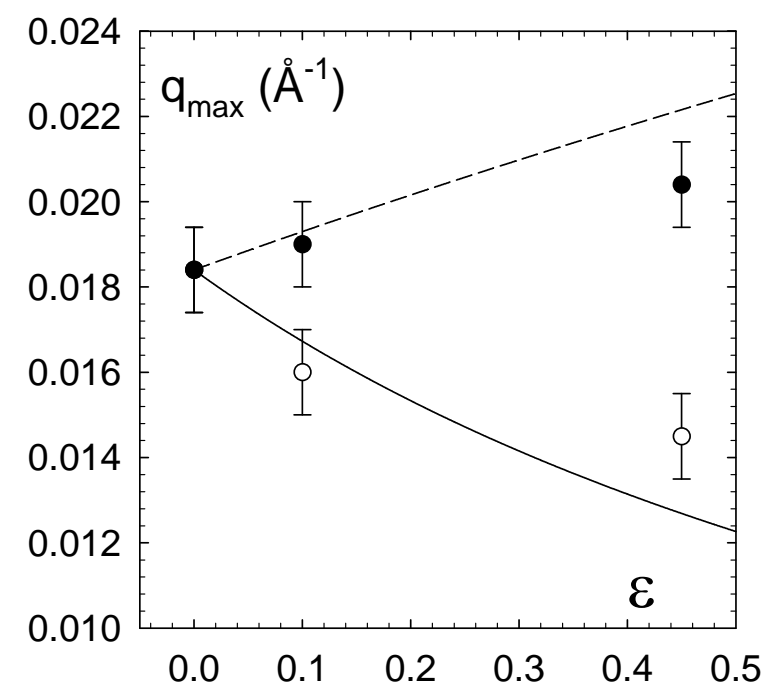

Figure 8

Strain dependence of $q_{\max }$ for SP5_PW0.14_R0.1 hydrogel in the parallel $(O)$ and perpendicular $(\mathbf{O})$ directions of stretching. The solid line and short-dash line are the theoretical plots calculated for affine deformation in the parallel direction and perpendicular one, respectively.

The same anisotropic scattering pattern has also been observed for SP2_PW0.14_R0.1 hybrid hydrogels under uniaxial stretching (see Figure S5 in supporting information). In that case, the double wing pattern is preserved at $\varepsilon=100 \%$ as the volume fraction of silica particles is about two times less compared to SP5 formulation. Nevertheless a four spot pattern is obtained at higher deformation $(\varepsilon=370 \%)$; i.e. well above the critical value roughly calculated from a simple cubic lattice arrangement: $\varepsilon_{c r} \cong 200 \%$. We can also notice that when the sample is maintained under high deformation $(\varepsilon=370 \%)$ during a long period (10 hours) there is mainly no change in the scattering pattern of the sample and consequently in the distribution of silica particles under large strain. This could be different for the polymer chains.

\section{Reversibility of anisotropic patterns}

In order to study the recovery of the samples, through the distribution of silica nanoparticles in the polymer matrix, loading/unloading experiments were carried out on samples SP2_PW0.14_R0.1, SP5_PW0.14_R0.1 and SP5_PW0.14_R1 at intermediate strains. The scattering spectra given in Figure 9 compare the samples R0.1: 1) initially unstrained, 2) 
stretched during one hour under uni-axial deformation (at $\varepsilon=100 \%$ ) and 3) just after unloading $(\varepsilon=0 \%)$.
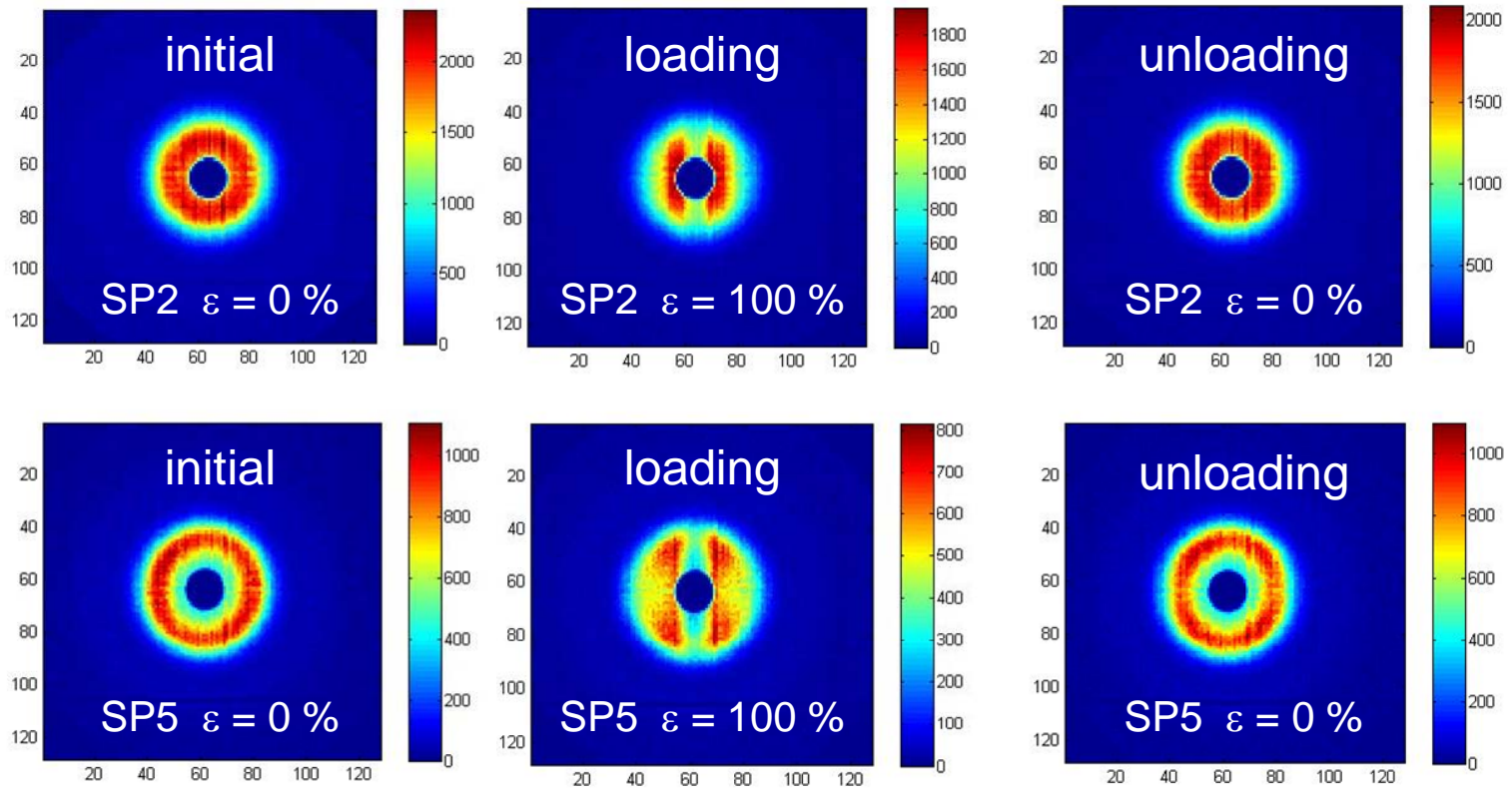

Figure 9: 2D-SANS patterns of SP2_PW0.14_R0.1 (up) and SP5_PW0.14_R0.1 (down) hybrid hydrogels before loading ( $\varepsilon=0 \%$; left), under loading (middle; $\varepsilon=100 \%$ ) and after unloading (right; $\varepsilon=0 \%$ ).

For SP2, we can notice that in these conditions the scattering from the silica nanoparticles recovers its initial isotropic pattern after unloading and we can reasonably assume that the same holds for the polymer matrix and the mechanical properties as well. A similar trend is observed for SP5_PW0.14_R1 when reversibly stretched during one hour at lower strain $(\varepsilon=$ 16\%; see Figure S6 in supporting information). By comparison with SP2_PW0.14_R0.1, for which the 2D image obtained after unloading seems similar to the initial one, SP5_PW0.14_R0.1 sample has kept a bit of four-spots pattern: it displays longer time dependence for its recovery. This can originate from the higher level of particles shearing induced by the transverse compression discussed previously. On the other hand, when the sample SP5_PW0.14_R0.1 is stretched well above its critical condition $(\varepsilon=200 \%)$ during half an hour and then unloaded back to $\varepsilon=45 \%$, the recovery is not complete and the four- 
spot pattern remains observable even after 3 hours (see supporting information Figure S7). In this case, where the sample has been highly deformed, the unloading was stopped at a strain level of $45 \%$ which corresponds to the deformation that is immediately recovered by the sample.

The main conclusion of this set of experiments is that silica particles embedded into the polymer matrix undergo various mechanical stresses under uniaxial deformation. Following the macroscopic deformation of the sample, the particles display affine motions at low strain but rapidly, the contact between particles and their polymer layer induced by transverse compression gives rise to non affine displacement with local shearing. The loading process appears to be reversible, on time scale shorter that it be probed by SANS, as long as the strain level is not too high and does not imply a high level of confinement of silica beads. For larger deformations, the particles do not recover their initial distribution, revealing the existence of some irreversible or long-term mechanisms.

\section{CONCLUSION}

We have shown in this work that it is possible to formulate nano-hybrid hydrogels on a large range of composition changing either the level of covalent cross-linker and/or the number of physical connectors formed by specific interactions between the matrix and silica nanoparticles (the silica particles). Whatever is the degree of permanent cross-links, all the networks show a dramatic improvement of their mechanical properties with the introduction of silica nano-particles as both stiffness and nominal strain at failure increase. As physical interactions are reversible in nature, the adsorption/desorption process of PDMA chains and consequently the mechanical reinforcement is time dependent. The mechanical behaviour of nano-hybrid hydrogels displays the possibility to tune the macroscopic properties by changing 
either the level of covalent cross-linker or the amount of added silica particles along with the time dependence of their interactions.

The SANS analysis of silica nano-particles performed by contrast matching experiments clearly evidence that the distribution of particles remains mostly unaffected by the polymerization of DMA in the presence of a low amount of chemical cross-linker $(\mathrm{R}=0$ to 1 mol\%). The spherical nano-particles display short range repulsive interactions that prevent them from aggregation during polymerization. Working at low degree of cross-linking $(\mathrm{R} \leq 1$ mol\%), in order to preserve a sufficient extensibility of the network, no significant impact of the SP ratio on the silica dispersion was evidenced; except at very low silica content (SP0.5) where a weak destabilization of the particles has been attributed to the lower $\mathrm{pH}$. This hypothesis underlines the key influence of the $\mathrm{pH}$ on nano-hybrid structures as it can readily impact the particle dispersion as well as the level of PDMA/silica interactions. At low $\mathrm{pH}$ for instance, Susuki et $a l^{[33]}$ have reported the aggregation behavior of silica particles of similar size within PNIPA hydrogels and similarly Drabarek et $a l^{[49]}$ have studied the sol-gel transition of Ludox particles at $\mathrm{pH} 8$ with the formation of percolating clusters with time. Although this aspect was out of scope in the present study, this demonstrates the high versatility of silica/PDMA formulations if $\mathrm{pH}$ and ionic strength are used as external parameters. It also points out the importance of controlling the aqueous environment of nanocomposite formulations during the hydrogel formation as it could strongly impact the final properties. Indeed, $\mathrm{pH}$ does not only modify the binding energy of polymer chains and adsorption properties at equilibrium but also the exchange dynamics. At $\mathrm{pH}$, where the fraction of silanol interacting groups is not very high, the adsorption of PDMA chains onto silica surfaces remains moderate and this could be the reason why PDMA chains do not form a very dense layer with immobilized chains at the silica interface. The formation of a dynamic polymer layer is then responsible for the time dependence of PDMA/silica interactions that 
can be easily observed by changing the deformation rate ${ }^{[19]}$ or waiting a few seconds to recover the residual strain for samples studied at low or intermediate strain. It is also the reason of the self-adhesive behavior of nano-hybrid hydrogels and we have shown recently that these hybrid interactions could find promising applications as silica nano-particles behave as efficient reversible adhesive not only for PDMA gels but also for other soft materials like biological tissues ${ }^{[50]}$. More generally, we believe that the dynamics of the adsorption/desorption process of polymer chains with the filler is a central feature in the reinforcement properties of nano-hybrid or nanocomposite hydrogels that deserve to be investigated in more details. By comparison with elastomer or polymer melt nanocomposites $^{[30,51]}$, several mechanisms have been proposed to explain reinforcement: 1) the formation of a glassy polymer layer in the vicinity of the fillers, 2) the filler aggregation and percolation giving rise to filler networking and 3) the increase of trapped entanglements around filler particles due to chain adsorption. From our results we can easily rule out hypotheses 1) and 2) as dynamics of interactions prevail on the mechanical properties and only a moderate destabilization of silica particles has been evidenced by SANS when increasing the level of chemical cross-links. As a consequence, we think that the peculiar mechanical behavior of nano-hybrid hydrogels arises from the formation of an amplified cross-linking density in the surroundings of the particles, induced by coupling covalent crosslinks and physical interactions between PDMA and silica.

Under deformation, the behavior of nano-hybrid hydrogels is similar to what has been reported with other soft hybrid materials: the particles move away in the parallel direction while they get closer in the transverse one. This rapidly gives rise to a non affine deformation of the hybrid network with shearing due to the transverse compression of the distance between particles. After unloading the sample initially stretched at intermediate deformation, the particles recover their initial structure at rest and we can assume that the restoring forces, 
coming from the polymer chains of the covalent network have not been damaged in these conditions. The situation is different for unloading after higher deformation for which we observe remaining orientation of the particles. This is attributed to a partial breakage of polymer chains or to some irreversible particle/particle adhesion during shearing.

\section{ACKNOWLEDGMENTS}

Ph.D. scholarship of Séverine Rose was provided by the Ph.D. School "Chemistry and Physics of Materials" of University Pierre and Marie Curie (ED 397, UPMC Paris, France). The authors are indebted to Costantino Creton and Ludwik Leibler for stimulating discussions and they also want to acknowledge David Martina (SIMM), Ludovic Olanier (SIMM) and Arnaud Hélary (LLB) for their help in the design of the deformation device of polymer gels for 2D SANS experiments.

\section{REFERENCES}

[1] N. A. Peppas, Hydrogels in Medicine and Pharmacy; CRC Press: Boca Raton, FL, 1986.

[2] F. L. Buchholz, A. T. Graham, Modern Superabsorbent Polymer Technology; WileyVCH: New-York, 1997.

[3] T. R. Hoare, D. S. Kohane, Polymer, 2008, 49, 1993-2007.

[4] J. K. Oh, R. Drumright, D. J. Siegwart, K. Matyjaszewski, Prog. Polym. Sci., 2008, 33, 448-477.

[5] J. P. Gong, Y. Katsuyama, T. Kurokawa, Y. Osada, Adv. Mater., 2003, 15, 1155-1158.

[6] Y. Okumura, K. Ito, Adv. Mater., 2001, 13, 485-487.

[7] T. Matsunaga, T. Sakai, Y. Akagi, U. I. Chung, M. Shibayama, Macromolecules, 2009, $42,6245-6252$.

[8] K. Haraguchi, T. Takehisa, S. Fan, Macromolecules, 2002, 35, 10162-10171.

[9] K. Haraguchi, R. Farnworth, A. Ohbayashi, T. Takehisa, Macromolecules, 2003, 36, $5732-5741$.

[10] J. Yang, X.-P. Wang, X.-M Xie, Soft Matter, 2012, 8, 1058-1063.

[11] Q. Wang, R. Hou, Y. Cheng, J. Fu, Soft Matter, 2012, 8, 6048-6056. 
[12] J. Yang, L.-H. Deng, C.-R. Han, J.-F. Duan, M.-G. Ma, X.-M. Zhang, F. Xuab, R.-C. Sunab, Soft Matter, 2013, 9, 1220-1230.

[13] A. Alam, M. Takafuji, H. Ihara, J. Of Colloid and Interface Science, 2013, 405, 109-117.

[14] D. Hourdet, L. Petit, Macromol. Symp., 2010, 291-292, 144-158.

[15] D. Portehault, L. Petit, N. Pantoustier, F. Lafuma, D. Hourdet, Coll. Surf. A, 2006, 78, 26-32.

[16] L. Petit, L. Bouteiller, A. Brûlet, F. Lafuma, D. Hourdet, Langmuir, 2007, 33, 147-158.

[17] L. Carlsson, S. Rose, D. Hourdet, A. Marcellan, Soft Matter, 2010, 6, 3619-3631.

[18] W. C. Lin, W. Fan, A. Marcellan, D. Hourdet, C. Creton, Macromolecules, 2010, 43, 2554-2563.

[19] S. Rose, A. Dizeux, T. Narita, D. Hourdet, A. Marcellan, Macromolecules, 2013, 46, 4095-4104.

[20] L. Petit, L. Carlsson, S. Rose, A. Marcellan, T. Narita, D. Hourdet, Macromol. Symp., 2014, 337, 58-73.

[21] R. S. Madabhushi, Electrophoresis, 1998, 19, 224-230.

[22] J. Sudor, V. Barbier, S. Thirot, D. Godfrin, D. Hourdet, M. Millequant, J. Blanchard, J.L. Viovy, Electrophoresis, 2001, 22, 720-728.

[23] L. Song, D. Liang, D. Fang, B. Chu, Electrophoresis, 2001, 22, 1987-1996.

[24] H. D. Bijsterbosch, M. A. Cohen Stuart, G. J. Fleer, Macromolecules, 1998, 31, 89818987.

[25] E. A. S. Doherty, K. D. Berglund, B. A. Buchholz, I. V. Kourkine, T. M. Przybycien, R. D. Tilton, A. E. Barron, Electrophoresis, 2002, 23, 2766-2776.

[26] P. Zhang, J. Ren, Analytica Chimica Acta, 2004, 507, 179-184.

[27] K. Haraguchi, H. J. Li, Macromolecules, 2006, 39, 1898-1905.

[28] Y. Liu, M. F. Zhu, X. L. Liu, W. Zhang, B. Sun, Y. M. Chen, H. J. P Adler, Polymer, 2006, 47, 1-5.

[29] Y. Y. Wu, M. G. Xia, Q. Q. Fan, Y. Zhang, H. Yu, M. F. Zhu, J. Polym. Sci. Pt. BPolym. Phys., 2011, 49, 263-266.

[30] S. S. Sternstein, A.-J. Zhu, Macromolecules, 2002, 35, 7262-7273.

[31] J. Yang, C.-R. Han, J.-F. Duan, F. Xu, R.-C. Sun, Nanoscale, 2013, 5, 10858-10863.

[32] G. Orts-Gil, K. Natte, D. Drescher, H. Bresch, A. Mantion, J. Kneipp, W. Osterle, J. Nanopart. Res., 2011, 13, 1593-1604.

[33] T. Suzuki, H. Endo, N. Osaka, M. Shibayama, Langmuir, 2009, 25, 8824-8832.

[34] A. P. Gast, L. Leibler, Journal of Physical Chemistry, 1985, 89, 3947-3949. 
[35] A. P. Gast, L. Leibler, Macromolecules, 1986, 19, 686-691.

[36] S. C. Lin, C. W. Whang, Journal of Separation Science, 2008, 31, 3921-3929.

[37] D. Qiu, C. Flood, T. Cosgrove, Langmuir, 2008, 24, 2983-2986.

[38] J. Bastide, L. Leibler, Macromolecules, 1988, 21, 2647-2649.

[39] M. Shibayama, H. Kurokawa, S. Nomura, S. Roy, R. S. Stein, W. L. Wu, Macromolecules, 1990, 23, 1438-1443.

[40] E. Mendes, R. Oeser, C. Hayes, F. Boué, J. Bastide, Macromolecules, 1996, 29, 55745584 .

[41] M. Shibayama, K. Kawakubo, F. Ikkai, M. Imai, Macromolecules, 1998, 31, 2586-2592.

[42] T. Karino, Y. Okumura, C. Zhao, T. Kataoka, K. Ito, M. Shibayama, Macromolecules, 2005, 38, 6161-6167.

[43] Y. Rharbi, B. Cabane, A. Vacher, M. Joanicot, F. Boué, Europhys. Lett., 1999, 46, 472478 .

[44] Y. Ikeda, Y. Yasuda, S. Yamamoto, Y. Morita, Journal of Applied Crystallography, 2007, 40, s549-s552.

[45] T. Nishida, H. Endo, N. Osaka, H.-J. Li, K. Haraguchi, M. Shibayama, Physical Review E, 2009, 80, 030801(1-4).

[46] S. Miyazaki, T. Karino, H. Endo, K. Haraguchi, M. Shibayama, Macromolecules, 2006, 39, 8112-8120.

[47] M. Shibayama, Polymer Journal, 2011, 43, 18-34.

[48] J. Oberdisse, Y. Rharbi, F. Boué, Computational and Theoretical Polymer Science, 2000, 10, 207-217.

[49] E. Drabarek, J. R. Bartlett, H. J. M. Hanley, J. L. Woolfrey, C. D. Muzny, International Journal of Thermophysics, 2002, 23, 145-160.

[50] S. Rose, A. Prevoteau, P. Elziere, D. Hourdet, A. Marcellan, L. Leibler, Nature, 2014, 505, 382-385.

[51] R. Pérez-Aparicio, A. Vieyres, P.-A. Albouy, O. Sanséau, L. Vanel, D. R. Long, P. Sotta, Macromolecules, 2013, 46, 8964-8972. 


\section{Supporting Information}

Structure investigation of nanohybrid PDMA/silica hydrogels at rest and under uniaxial deformation.

Séverine Rose ${ }^{1}$, Alba Marcellan ${ }^{1}$, Tetsuharu Narita ${ }^{1}$, François Boué ${ }^{2}$, Fabrice Cousin $^{2}$ and Dominique Hourdet ${ }^{1}$ *

${ }^{1}$ ESPCI / Sorbonne Universités, UPMC Univ Paris 06 / CNRS, UMR 7615, SIMM, 10 rue Vauquelin, F-75005, Paris, France

${ }^{2}$ Laboratoire Léon Brillouin, CEA-CNRS, Saclay 91191 Gif-sur-Yvette Cedex, France

\section{Small Angle Neutron Scattering (SANS)}

Experimental part

In order to investigate the contribution of both organic and inorganic components within hybrid hydrogels by SANS, contrast matching has been carried out using suitable $\mathrm{H}_{2} \mathrm{O} / \mathrm{D}_{2} \mathrm{O}$ ratios. Taking into account the scattering length densities of $\mathrm{H}_{2} \mathrm{O}\left(\rho_{\mathrm{H} 20}=-0.56 .10^{-6} \AA^{-2}\right)$, PDMA $\left(\rho_{\text {PDMA }}=0.936 .10^{-6} \AA^{-2}\right)$, silica $\left(\rho_{\mathrm{f}}=3.48 .10^{-6} \AA^{-2}\right)$ and $\mathrm{D}_{2} \mathrm{O}\left(\rho_{\mathrm{D} 20}=6.41 .10^{-6} \AA^{-2}\right)$, the contrast matching of PDMA and silica can be achieved with the following volume ratios:

1) $\mathrm{H}_{2} \mathrm{O} / \mathrm{D}_{2} \mathrm{O}=0.79 / 0.21$, named "solvent $\mathrm{p}$ " for matching the PDMA network ( $\mathrm{p}=$ polymer),

2) $\mathrm{H}_{2} \mathrm{O} / \mathrm{D}_{2} \mathrm{O}=0.42 / 0.58$, named "solvent $\mathrm{f}$ " for matching the silica nanoparticles ( $\mathrm{f}=$ filler).

For that purpose, hybrid hydrogels were specially prepared, following the same general procedure, in three different aqueous environments: solvent $p$, solvent $\mathrm{f}$ and pure $\mathrm{H}_{2} \mathrm{O}$. For hybrid hydrogels with high amounts of $\mathrm{D}_{2} \mathrm{O}$, Ludox-TM50 was initially dialysed during two weeks in control $\mathrm{D}_{2} \mathrm{O}$ environment in order to get a stable and concentrated dispersion of silica in $\mathrm{D}_{2} \mathrm{O}$ (around $40 \mathrm{wt} \%$ ), with $\mathrm{pH} \cong 9\left(\mathrm{pH}=8.6\right.$ as measured in $\left.\mathrm{D}_{2} \mathrm{O}\right)$ and a conductivity of $4.9 \mathrm{mS} / \mathrm{cm}$.

In order to perform SANS experiments on gel samples under controlled deformation and environment, a special cell has been designed in collaboration with the Laboratoire Léon Brillouin. The main specifications of the cell (see Figure S1), which is inserted in the automatic sample changer of the PAXY spectrometer, are the following:

- an efficient fixation setup for the gel sample that prevents sliding,

- an accurate control in situ of the sample elongation with micrometric screws,

- a tight fit cell with a fine control of the environment, such as temperature, solvent or humidity,

- quartz windows, tightly closed and transparent for neutrons, with a sufficient diameter for the neutrons beam (about $7.3 \mathrm{~mm}$ ). 

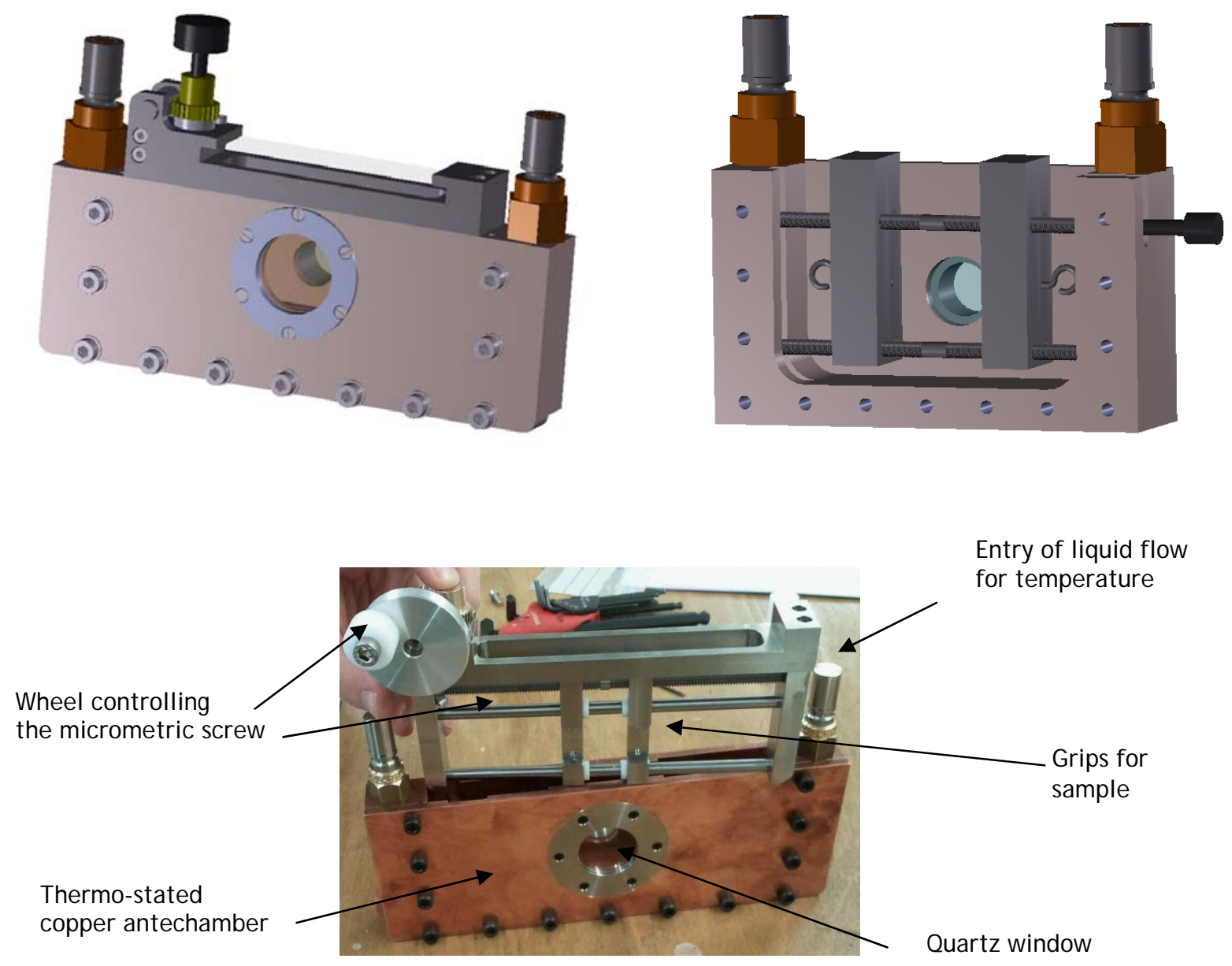

Figure S1: Up: Schematic representations of the 2D SANS device, with top left: the entire device, and top right: an internal view.

Down: picture of the experimental device with legends.

In the case of hybrid hydrogels containing large amounts of water, a long stay in air induces drying that modifies the network properties (concentration, elastic modulus, etc...). This problem becomes critical with anisotropic analyses (longer acquisition times) performed on gel samples submitted to successive deformation states which could last for hours. For that reason, all anisotropic experiments on gel samples in their preparation state were carried out in immersed conditions; the cell chamber being filled with a fluorinated oil highly transparent for neutrons (perfluorodecalin). No interaction between hybrid hydrogels and fluorinated oil was observed in these conditions and hydrogels remained stable with time for hours, even days. In 2D SANS experiments, the incident neutron flux recorded on a two dimensional detector built up with 15500 cells of $25 \mathrm{~mm}^{2}$, is directly transformed into a 2D image with a colour code. After sector averaging, the data can be quantitatively analyzed in terms of diffusion pattern. 


\section{$\underline{\text { SANS analysis of dilute dispersion of Ludox TM-50 }}$}

In dilute condition, when interactions between particles are negligible, $S(q)=1$ over the whole range of q and $I_{c o r}(q)$ is equal to the form factor. An example is given in Figure S2 with a dilute dispersion of Ludox TM50 (1 wt\%) along with the fit obtained from the polydisperse sphere model. Even if the reduced scattering intensity, equal in these conditions to the form factor, is very noisy at large q after subtraction of the solvent, this first set of data allows a good assessment of the particle size; their volume being extrapolated at $q \rightarrow 0: \mathrm{I}_{\text {cor }}(0)$ $=\mathrm{P}(0)=V_{f} \cong 10^{7} \AA^{3}$. The best fitting is obtained with a particle radius $R_{f}=132 \AA$ and a standard deviation $\sigma=15 \AA$. These results are in good agreement with those obtained from other techniques, either in the dry state by SEM $\left(R_{f}=140-150 \AA\right.$ Figure S2 $)$ or in the dispersed state by DLS (170 ̊).

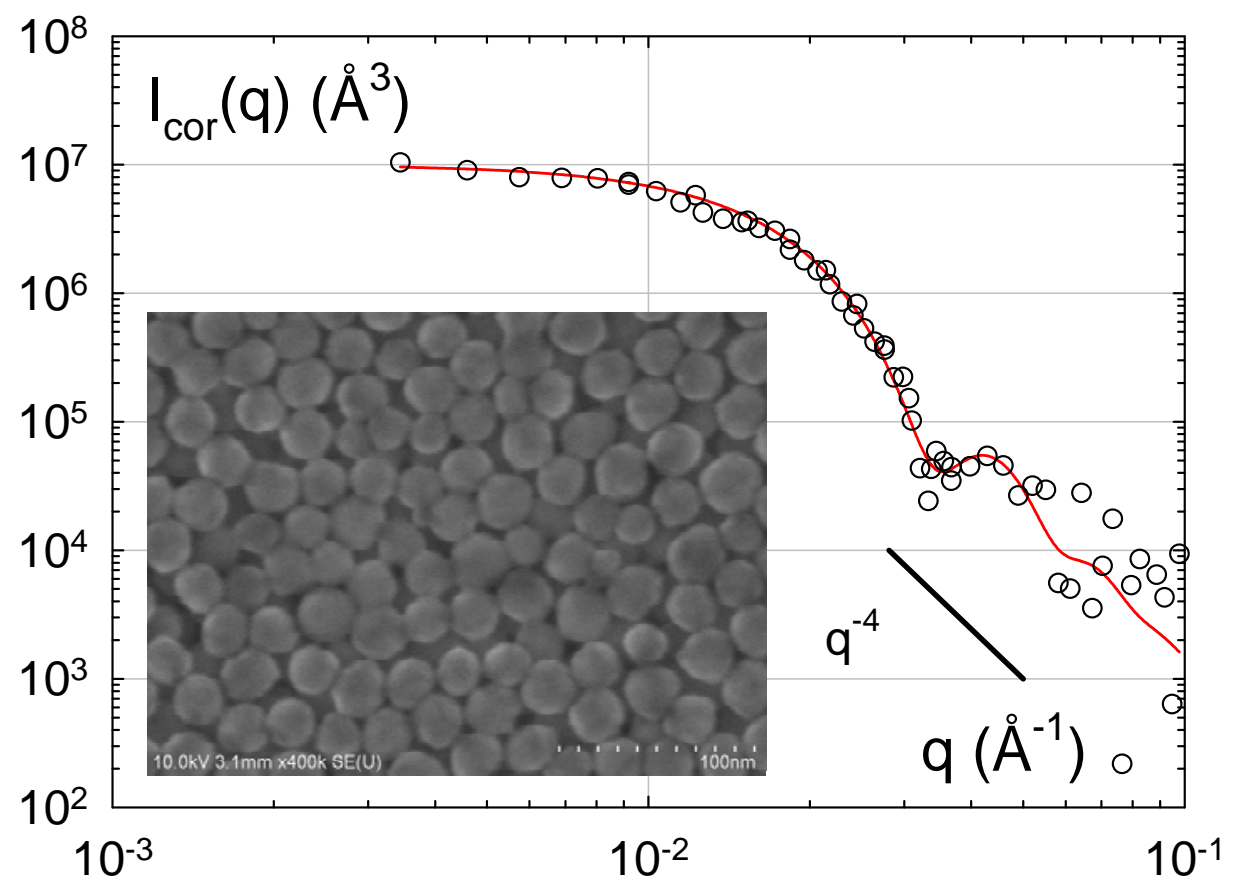

Figure S2. Characterization of silica nanoparticles Ludox TM50 by SANS with a double logarithmic plot of the reduced scattering intensity $(O)$ obtained from a dilute dispersion of silica nanoparticles $(C=1 \mathrm{wt} \%)$; the corresponding fit, based on a polydisperse sphere model $\left(R_{f}=132 \AA\right.$ and $\left.\sigma=15 \AA\right)$, is indicated in solid line. The SEM image of silica nanoparticles is given in the inset $\left(R_{f}=140-150 \AA\right)$.

These results also agree with numerous characterization reported on Ludox TM50 in the literature $^{[1-3]}$. As discussed by Orts-Gill and co-workers, which obtained similar values by SAXS, TEM and DLS, the hydrodynamic radius obtained by DLS is generally overestimated in dilute solution due i) to larger objects that contribute to higher intensity and ii) to the increasing particles repulsions observed by dilution ${ }^{[3]}$. 


\section{$\underline{\text { SANS fitting for concentrated dispersions }}$}

In order to model the scattering behaviour of silica dispersions and hybrid hydrogels with a minimum number of adjustable parameters, a hard sphere potential between particles has been assumed. Considering the Percus-Yevick approximation of the Ornstein-Zernike equation, the structure factor for hard spheres can be calculated using the following set of equations:

$$
S(q)=\frac{1}{1+\frac{24 \phi_{H S} G\left(2 q R_{H S}\right)}{2 q R_{H S}}}
$$

with $R_{H S}$ the radius of hard spheres with volume fraction $\phi_{H S}$, and $G$ a trigonometric function depending on $q, R_{H S}$ and $\phi_{H S}$ as follows:

$$
\begin{aligned}
& G(x)=\alpha \frac{(\sin x-x \cos x)}{x^{2}}+\beta \frac{2 x \sin x+\left(2-x^{2}\right) \cos x-2}{x^{3}} \\
& +\gamma \frac{\left\{-x^{4} \cos x+4\left[\left(3 x^{2}-6\right) \cos x+\left(x^{3}-6 x\right) \sin x+6\right]\right\}}{x^{5}}
\end{aligned}
$$

with $\quad \alpha=\frac{\left(1+2 \phi_{H S}\right)^{2}}{\left(1-\phi_{H S}\right)^{4}} \quad \beta=-6 \phi_{H S} \frac{\left(1+\left(\phi_{H S} / 2\right)\right)^{2}}{\left(1-\phi_{H S}\right)^{4}} \quad \gamma=\frac{\left(\phi_{H S} / 2\right)\left(1+2 \phi_{H S}\right)^{2}}{\left(1-\phi_{H S}\right)^{4}}$

Working with absolute scattering intensities and known formulation parameters like the volume fraction of silica particles $\left(\phi_{f}\right)$ and the nature of the diluent, it is possible to fit the experimental data according to equation $\{8\}$ by using a simple set of unequivocal variables: $R, \sigma$ and $R_{H S} ; \phi_{H S}$ being given by $\phi_{H S}=\phi_{f}\left(R_{H S} / R\right)^{3}$.

An example is given in Figure S3. 


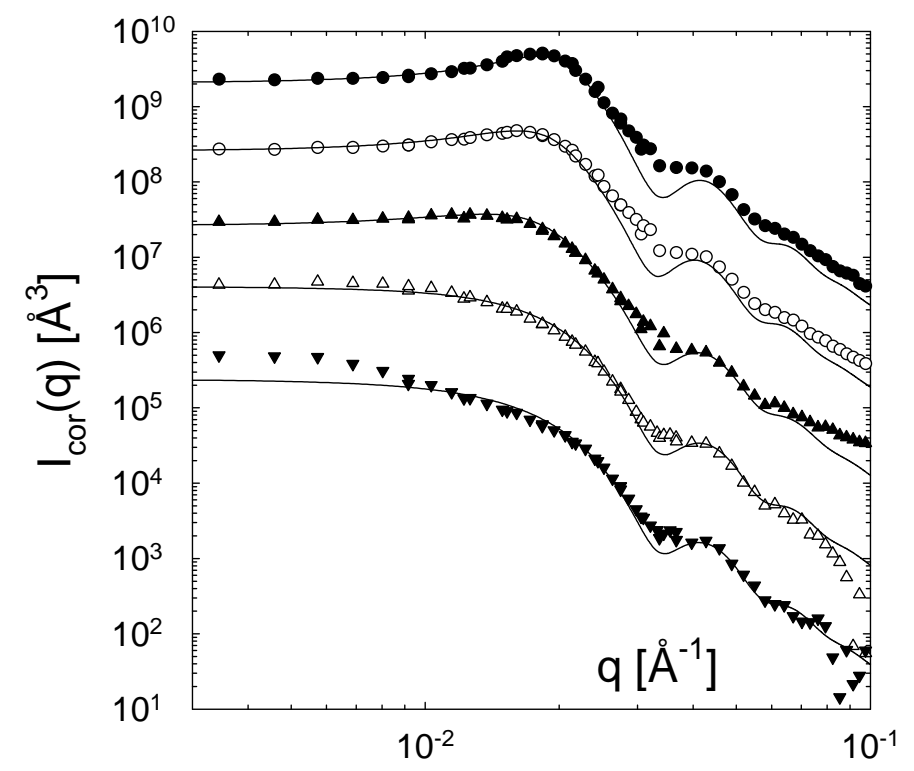

Figure S3

Double logarithmic plots of the normalized scattered intensity of PDMA/silica hydrogels (SPx_PW0.14_R0.1) prepared in solvent $p$ (silica scattering) with different amount of silica: experimental data for SP0.5 ( $)$, SP1 $(\triangle)$, SP2 ( $\mathbf{\Delta})$, SP3.5 (O), SP4.8 (O) and model fitting. The curves are shifted from one to another by a factor 10; the bottom one ( $\boldsymbol{\nabla})$ being the reference position.

\section{Analysis of the corona}

Working in solvent $\mathrm{f}$, the filler and the solvent have the same scattering length densities $\left(\rho_{s}=\rho_{f}\right)$ and the situation is similar to a PDMA hydrogel with solvent holes replacing the silica nanoparticles. In this case, we can consider a 3-phase model with: 1) the filler (index f), 2) the adsorbed polymer layer at the interface (index $l$ ) and 3) the PDMA matrix which does not interact directly with the particles (index $m$ ).

In order to take into account this situation we have used the form factor describing the scattering of the hard sphere interface between the core and the corona (adsorbed polymer layer) and between the corona and the surrounding polymer matrix. The scattering amplitude of the core-shell particle is given by ${ }^{[4]}$ :

$$
\begin{aligned}
A(q) & =V_{l}\left(\rho_{l}-\rho_{m}\right) F\left(q, R_{l}\right)+V_{f}\left(\rho_{f}-\rho_{l}\right) F\left(q, R_{f}\right) \quad\{\mathrm{S} 4\} \\
\text { or } \quad A(q) & =\left(\rho_{p}-\rho_{s}\right)\left\{\left(\phi_{l}-\phi_{m}\right) V_{l} F\left(q, R_{l}\right)-\phi_{l} V_{f} F\left(q, R_{f}\right)\right\} \quad\{\mathrm{S} 5
\end{aligned}
$$

with $\phi_{l}$ and $\phi_{m}$ the volume fraction of polymer in the layer and out of the layer, $F\left(q, R_{i}\right)$ the scattering amplitude of spheres of radius $R_{i}$ defined by $\{$ Eq. 10$\}$ in the manuscript, $V_{i}=4 \pi R_{i}^{3} / 3$ the volume of spheres with $R_{l}=R_{f}+t$ where $\mathrm{t}$ is the thickness of the adsorbed polymer layer(see Figure S4a). 
The impact of the polymer layer on the amplitude of the form factor is emphasized in Figure S4b where the thickness and/or the concentration of polymer have been varied.

A we can see, these modifications involve dramatic changes of scattering patterns due to i) large variations of the scattering volume $\left(\{A(0)\}^{2}=\left\{V_{l}\left(\rho_{l}-\rho_{m}\right)+V_{f}\left(\rho_{f}-\rho_{l}\right)\right\}^{2}\right)$ as a function of the characteristics of the polymer layer (thickness and concentration) and ii) interferences between the core and the shell that give rise to additional oscillations in the scattering spectrum.
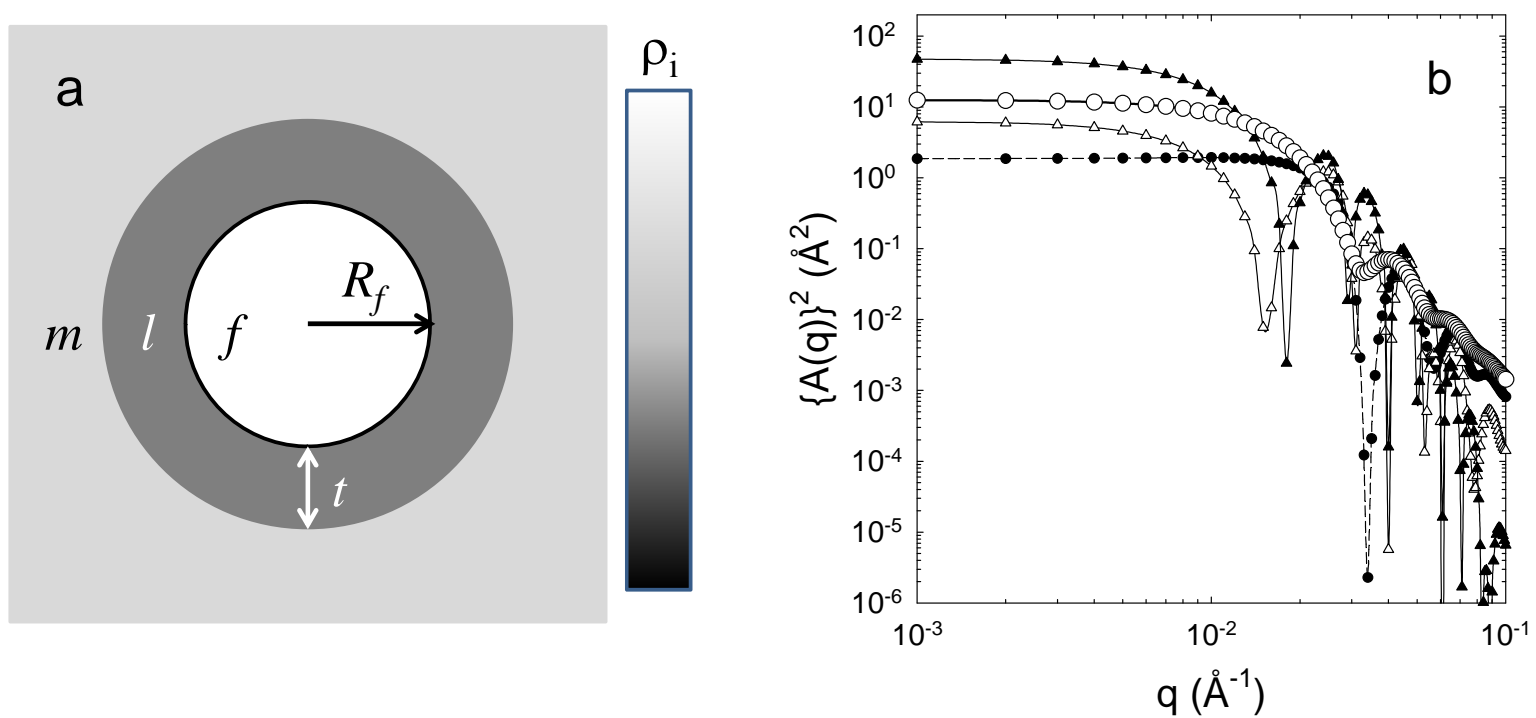

Figure S4. (a) Schematic representation of the structure of hybrid network with the silica core (index $f$ ), the adsorbed polymer layer at the interface (index $l$ ) and the polymer matrix (index $m$ ). The grey scale is used to qualitatively picture the level of scattering length densities in the different domains. (b) Variation of the square of the scattering amplitude of core-shell particles for various concentration and thickness of the polymer layer $\left(R_{f}=135 \AA\right.$ and $\sigma=$ 15): $\mathrm{t}=0 \AA$ with $\phi_{1}=\phi_{\mathrm{p}}=0.124(\mathrm{O}) ; \mathrm{t}=20 \AA$ with $\phi_{1}=0.25$ and $\phi_{\mathrm{p}}=0.116(\bigcirc) ; \mathrm{t}=20 \AA$ and $\phi_{1}=0.50$ and $\phi_{\mathrm{p}}=0.101(\triangle) ; \mathrm{t}=30 \AA$ and $\phi_{\mathrm{l}}=0.50$ and $\phi_{\mathrm{p}}=0.070(\boldsymbol{\Delta})$.

\section{D SANS}

Under uniaxial stretching, an anisotropic scattering pattern is observed for SP2_PW0.14_R0.1

hybrid hydrogel. As shown in Figure S5, the double wing patterns is preserved at $\varepsilon=100 \%$ as the volume fraction of silica particles is about two times less compared to SP5 formulation. Nevertheless a four spot pattern is obtained at higher deformation $(\varepsilon=370 \%)$; i.e. well above the critical value roughly calculated from a simple cubic lattice arrangement: $\varepsilon_{c r} \cong 200 \%$. We can also notice that when the sample is maintained under high deformation $(\varepsilon=370 \%)$ 
during a long period (10 hours) there is mainly no change in the scattering pattern of the sample and consequently in the distribution of silica particles under large strain.
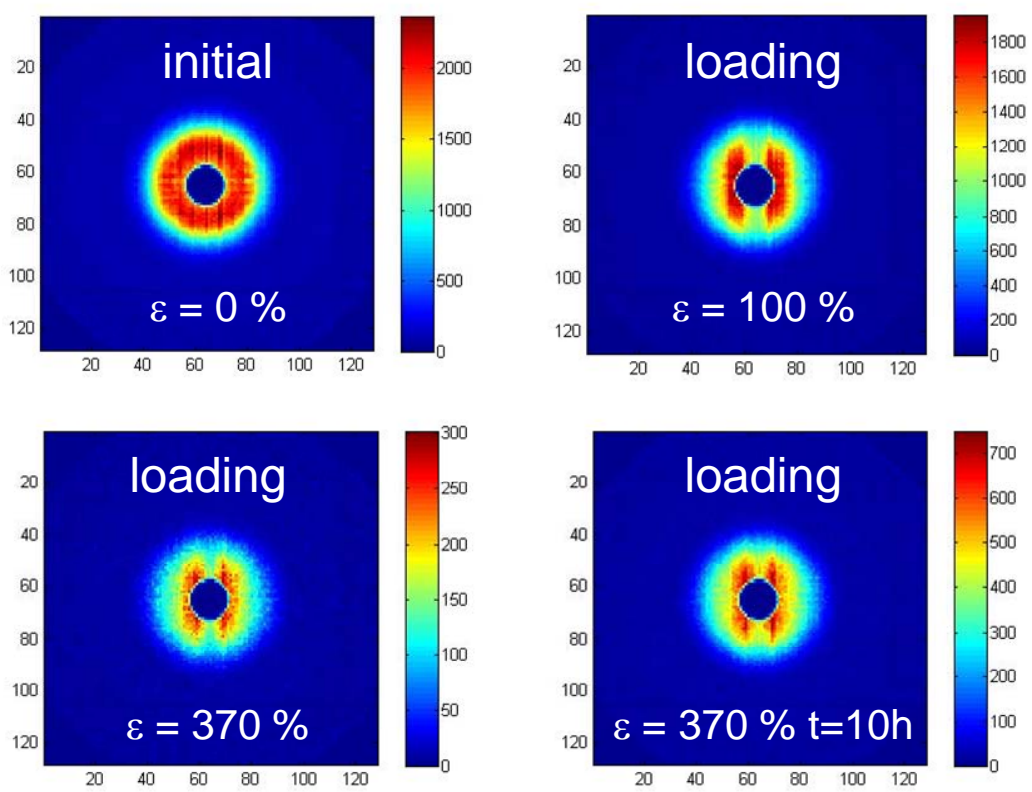

Figure S5: 2D-SANS images of SP2 PW0.14 R0.1 hybrid hydrogel. Top left: unstrained sample $(\varepsilon=0 \%)$; top right $(\varepsilon=100 \%)$; bottom left $(\varepsilon=370 \%)$; bottom right $(\varepsilon=370 \%)$ after 10 hours loading at this strain.

When stretched at relatively low deformation $(\varepsilon=16 \%)$, the SP5_PW0.14_R1 hydrogel fully recovered its isotropic behaviour after unloading (Figure S6).
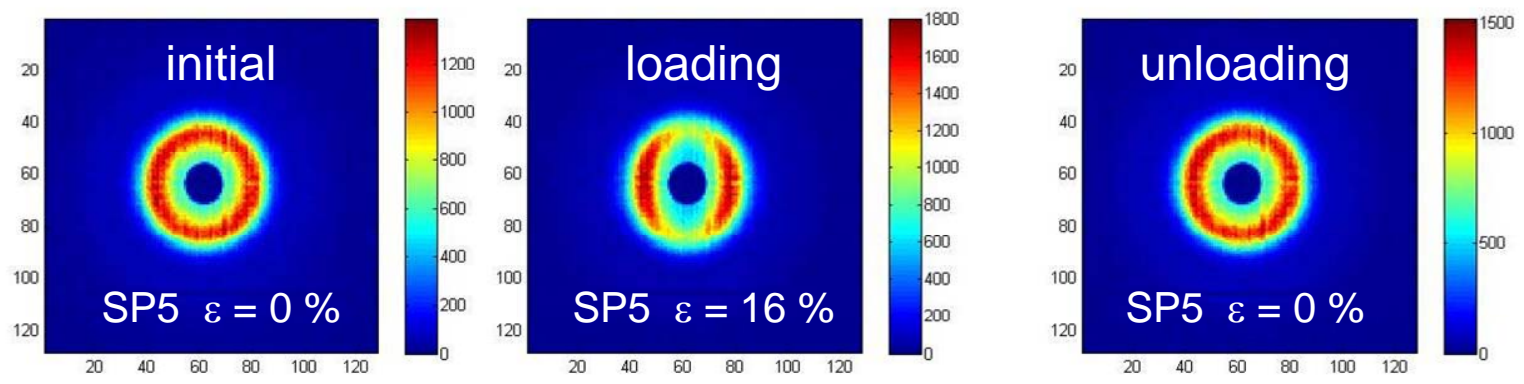

Figure S6: 2D-SANS patterns of SP5_PW0.14_R1 hybrid hydrogel before loading (left; $\varepsilon=0 \%$ )), under loading (middle; $\varepsilon=16 \%$ ) and after unloading (right; $\varepsilon=0 \%$ ).

On the other hand, when the sample SP5_PW0.14_R0.1 is stretched well above its critical condition ( $\varepsilon=200 \%$ ) during half an hour and then unloaded back to $\varepsilon=45 \%$, the recovery is not complete and the four-spot pattern remains observable even after 3 hours (Figure S7). 


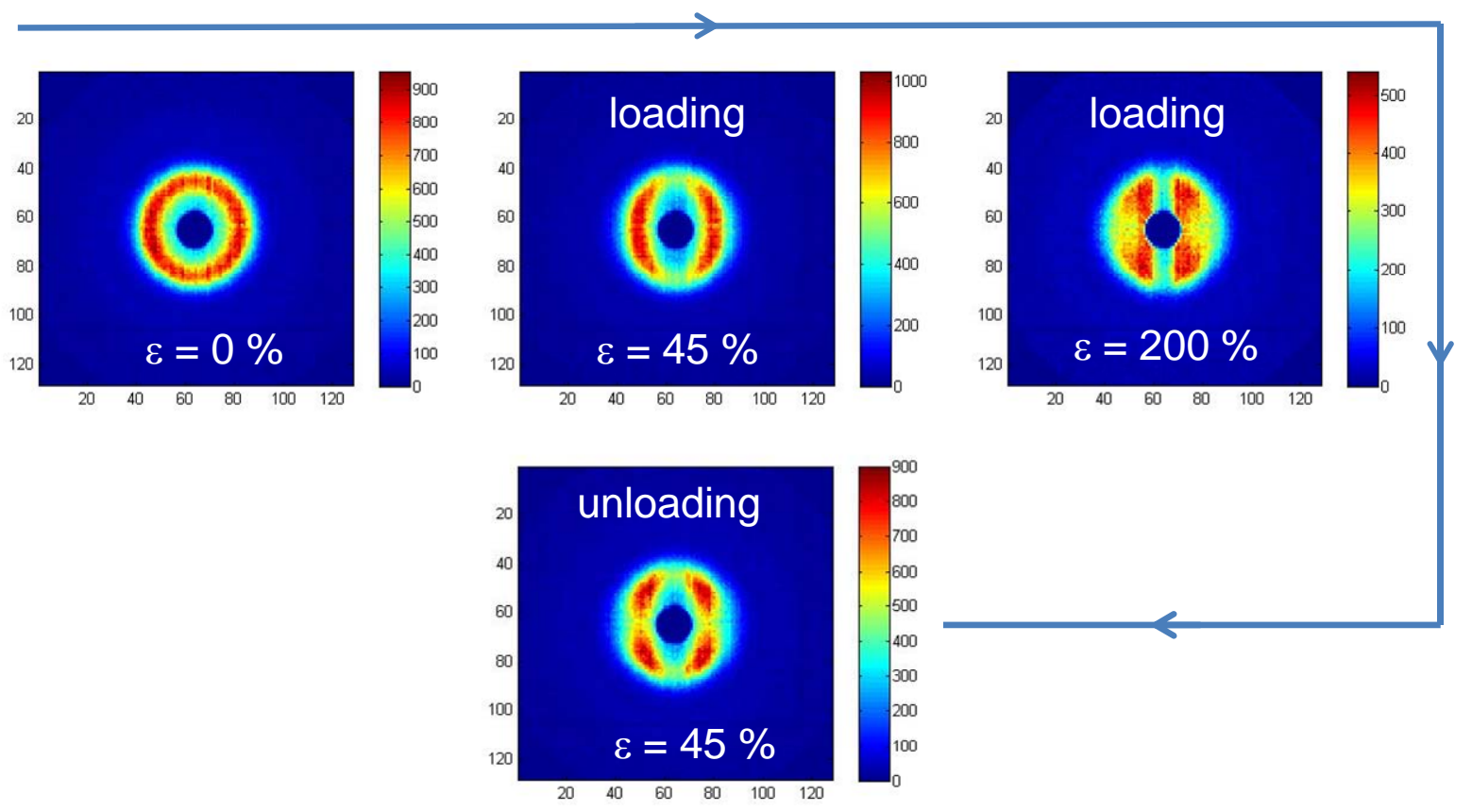

Figure S7: 2D-SANS patterns of SP5_PW0.14_R0.1 hybrid hydrogel. Top left: unstrained sample $(\varepsilon=0 \%)$; top middle and top right: loading at $\varepsilon=45 \%$ and then $200 \%$; bottom: unloading from $\varepsilon=200 \%$ to $\varepsilon=45 \%$.

\section{Reference}

[1] V. Goertz, N. Dingemouts, H. Nirschl, Part. Part. Syst. Charact., 2009, 26, 17-24.

[2] N. Hould, R. Lobo, N. Wagner, Part. Part. Syst. Charact., 2010, 27, 89-99.

[3] G. Orts-Gil, K. Natte, D. Drescher, H. Bresch, A. Mantion, J. Kneipp, W. Osterle, J. Nanopart. Res., 2011, 13, 1593-1604.

[4] Suzuki, T.; Endo, H.; Osaka, N.; Shibayama, M. Langmuir 2009, 25, 8824-8832. 